\title{
A numerical analysis of allocation strategies for the multi-armed bandit problem under delayed rewards conditions in digital campaign management ${ }^{\text {is }}$ Miguel Martín, Antonio Jiménez-Martín *, Alfonso Mateos \\ Departamento de Inteligencia Artificial, Universidad Politécnica de Madrid, Campus de Montegancedo S/N, Boadilla del Monte Madrid 28660, Spain
}

\section{A B S T R A C T}

In this paper, we analyze the most representative allocation strategies to deal with the multi-armed ban- dit problem in a context with delayed rewards by means of a numerical study based on a discrete event simulation. The scenario that we address is a digital marketing content recommendation system, called campaign management, used by marketers to create specific digital content that can be issued or config- ured for viewing by certain population segments according to a series of business variables, user profile or behavior. Both batch mode and online update architectures are considered for feedback from the dif- ferent contents displayed to users. The results show that possibilistic reward (PR) methods outperform other allocation strategies in this scenario with delayed rewards.

\section{Introduction}

The multi-armed bandit problem has been studied in great depth in statistics [7], where it has become central to different areas of economics, statistics or artificial intelligence [4,16], such as reinforcement learning [24] and evolutionary programming [12].

The name bandit stems from the image of a gambler playing with $K$ slot machines. The gambler can pull the arm of any of the machines, which produces a reward payoff. Since the reward distributions are initially unknown, the gambler must use exploratory actions to learn the utility of the individual arms. However, exploration has to be controlled since excessive exploration may lead to unnecessary losses. Thus, the gambler must carefully balance exploration and exploitation.

A gambler learning the distributions of arm rewards can use all past information to decide about his next action. Therefore, a policy or allocation strategy is an algorithm that chooses the next arm to play based on the sequence of previous plays and resulting rewards. The goal is to maximize the sum of the rewards received or equivalently to minimize regret. Regret is defined as the loss

\footnotetext{
The paper was supported by Spanish Ministry of Economy and Competitiveness projects MTM2014-56949-C3-2-R and MTM2017-86875-C3-3R.

* Corresponding author.

E-mail addresses: miguel.martin@alumnos.upm.es (M. Martín) antonio.jimenez@upm.es (A. Jiménez-Martín), alfonso.mateos@upm.es (A. Mateos).
}

compared to the total reward that can be achieved given full knowledge of the problem, i.e., when the arm giving the highest expected reward is pulled/played all the time.

There are two families of bandit settings [11]. In the first, the distribution of $X_{i t}$ is assumed to belong to a family of probability distributions $\left\{p_{\theta}, \theta \in \Theta_{i}\right\}$, whereas, in the second, the rewards are assumed to be bounded (say, between 0 and 1), and policies rely directly on the estimates of the expected rewards for each arm.

Almost all the policies or allocation strategies in the literature focus on the first family, and they can be separated into two distinct approaches [15]: the frequentist view and the Bayesian approach. In the frequentist view, the expected mean rewards for all arms are considered as unknown deterministic quantities and the goal of the algorithm is to reach the best parameter-dependent performance, whereas the parameter is drawn from a prior distribution instead of considering a deterministic unknown quantity in the Bayesian perspective. Bayesian performance is then defined as the average performance over all possible problem instances weighted by the prior on the parameters.

Another family of algorithms for solving bandit problems is so-called Thompson sampling (TS) [25], consisting of randomly drawing each arm according to its probability of being optimal.

A review of the most important allocation strategies belonging to the above bandit settings can be found in [20], and a numerical study on the basis of five complex and representative scenarios was performed in [21] to compare their performances. 
Other variants of the multi-armed bandit (MAB) problem (bandits with side information, bandits with no stochastic rewards, bandits with budgeted cost allocations...) can also be found in the literature $[8,18,19,26,27]$.

The allocation strategies analyzed in [21] do not account for delayed rewards. However, the reward for choosing one or the other action in real situations, such as Internet marketing advertising, clinical trials or content recommenders, is usually received with a delay after the time at which the action was executed, giving the algorithm the chance to work in the period from when the action is taken and the respective reward is received and pick the actions to be taken in subsequent iterations without having to update its strategy.

Joulani et al. [14] analyze the effect of the delay on the regret of online learning algorithms, concluding that delay additively increases the regret in stochastic problems. Moreover, they also propose the QPM-D algorithm that enacts a black-box transformation of algorithms developed for the non-delayed case into algorithms that can handle the presence of delays, including modifications of the UCB algorithm for the bandit problem with delayed feedback.

The so-called bandits with delayed, aggregated anonymous feedback are studied in [23]. At the end of each round, the player observes only the sum of a number of previously generated rewards that happen to arrive in the given round. The rewards are stochastically delayed, and, because the observations are aggregated, the information on which arm led to a particular reward is lost. Note that this is not the delay scenario considered in this paper, where rewards are not aggregated.

In this paper, we conduct a numerical analysis to analyze the performance of the most important allocation strategies in the literature for the MAB problem in a scenario with delayed rewards in a specific digital marketing content recommendation system for campaign management, aimed at personalizing the web site contents of a company. We account for two types of campaign management architectures when dealing with how to update the feedback of the different contents displayed to users, architectures with batch mode and online update.

To do this, we implement discrete event simulation to account for both an adaptation of the QPM-D algorithm proposed in [14] for the MAB problem and for the original MAB allocation strategies. We consider different reward distributions and stochastic delays, representing different digital campaign management scenarios within online and batch mode update architectures.

The paper is structured as follows. Section 2 gives a brief description of the allocation strategies for the MAB problem under consideration together with the proposed discrete event simulation to account for delayed rewards. Section 3 provides further information about the digital marketing content recommendation system used as a scenario in the simulation process, called campaign management. Section 4 describes the numerical analysis carried out and the results. Finally, some conclusions and future research work are outlined in Section 5.

\section{Allocation strategies for the MAB problem with delayed rewards}

The analysis conducted in this paper accounts for the most representative allocation strategies in the literature to deal with the MAB problem using logarithmic asymptotic convergence, thus discarding greedy, $\epsilon$ - greedy, and similar methods.

Table 1 shows the allocation strategies, together with information about their type, references in which they were introduced and whether or not they perform stochastic arm selection.

Lai and Robbins [17] first constructed a theoretical framework for determining optimal policies from the frequentist perspective. These policies work by associating a quantity called upper confidence index to each arm. This index relies on the entire sequence of rewards received so far from a given arm. Agrawal [1] introduced a generic class of index policies termed upper confidence bounds (UCB), where the index can be expressed as a simple function of the total reward received so far from the arm. Since then, different policies based on UCB have been reported in the literature. Auer et al. [5] strengthen previous results by showing easy-to-implement and computationally efficient policies (UCB1, UCB2 and UCB-Tuned) that achieve logarithmic regret uniformly over time rather than just asymptotically.

The Kullback-Leibler (KL)-based algorithms also belong to the frequentist perspective. The deterministic minimum empirical divergence (DMED) policy [13] was inspired by a Bayesian approach for the problem (although a Bayesian framework is not used for theoretical analyses). Later, the KL-UCB algorithm and its variant KL-UCB+ were introduced [11]. KL-UCB satisfied a uniformly better regret bound than UCB and its variants for arbitrary bounded rewards, whereas it reached the Lai and Robbins' lower bound with respect to Bernoulli rewards.

The BESA algorithm [6] is not based on the computation of an empirical confidence bounds, nor can it be classified as a KL-based algorithm. BESA is fully non- parametric.

The Thompson sampling (TS) algorithm [25] consists of randomly drawing each arm according to its probability of being optimal. The algorithm assumes that the arms' distributions belong to a parametric family of distributions $P=\{p(. \mid \theta), \theta \in \Theta\}$, where $\Theta \subseteq R$. It starts by putting a prior distribution on each one of the arms' parameters, maintaining at each time step a posterior distribution according to the rewards observed so far.

Finally, the possibilistic reward (PR) methods in $[20,21]$ were the first to use possibilistic reward distributions to model the uncertainty about the expected rewards from the arm, derived from a set of infinite confidence intervals nested around the expected value. Next, they use a pignistic probability transformation to convert these possibilistic functions into probability distributions following the insufficient reason principle and. Finally, Thompson sampling techniques are used to identify the arm with the highest expected reward and play the arm.

Depending on the inequality used to compute the confidence intervals, there are three possible PR methods with different

Table 1

Allocation strategy features.

\begin{tabular}{llll}
\hline Method & Type & Reference & Is Stochastic arm selection performed? \\
\hline UCB & Upper confidence bounds & Agrawal [1], Auer et al. [5] & No \\
DMED & Kullback-Leibler-based algorithms & Honda and Takemura [13] & No \\
KL-UCB & Kullback-Leibler-based algorithms & Garivier and Cappé [11] & No \\
KL-UCB + & Kullback-Leibler-based algorithms & Garivier and Cappé [11] & No \\
BESA & Non-parametric algorithm & Baransi et al. [6] & Yes \\
TS & Thompson sampling & Thompson [25] & Yes \\
PR1 & Possibilistic reward & Martín et al. [20] & Yes \\
PR3 & Possibilistic reward & Martín et al. [21] & Yes \\
\hline
\end{tabular}


features: PR1 and PR2, based on the Hoeffdings extension of the Chernoff bound, and PR3, which combines the Hoeffdings extension of the Chernoff bound and Bernstein bound.

As pointed out in [21], TS, PR2 and PR3 are the same in the case of a Bernoulli distribution for the arm rewards. Besides, PR2 is exactly the same as the generalization of the TS method proposed in [3] (see Algorithm 2).

The above allocation strategies do not originally account for delayed rewards. However, as mentioned before, the reward for choosing one or the other action in real situations, such as Internet marketing advertising, clinical trials or content recommenders, is usually received with a delay after the time at which the action was executed, giving the algorithm the chance to work in the period from when the action is taken and the respective reward is received and pick the actions to be taken in subsequent iterations without having to update its strategy.

The effect of delayed feedback has been studied in the literature within different online learning scenarios and different delay configurations, for both adversarial ([2,22]) and stochastic feedback. A concise summary including the (expected) regret in the delayed settings is given in [14].

Considering a fixed and known delay, Dudik et al. [10] showed an additive penalty in the regret for the stochastic setting (with side information). The problem of delayed feedback has also been studied for Gaussian process bandit optimization [9], resulting in a multiplicative increase in the regret that is independent of the delay and an additive term depending on the maximum delay.

Joulani et al. [14] provide black-box algorithms for delayed feedback in both the adversarial and the finite stochastic settings, assuming that there is a base allocation strategy (BASE) for solving the prediction problem without delay. The queued partial monitoring with delayed feedback (QPM-D) algorithm is proposed in [14] to deal with partial monitoring rather than bandit feedback for a finite stochastic setting.

\subsection{Discrete event simulation}

In this paper, we propose applying discrete event simulation to both an adaptation of the QPM-D algorithm for the MAB problem and the original MAB allocation strategies.

We consider two types of events in the discrete event simulation: the reception of a delayed reward and the selection of an arm based on the base allocation strategy (BASE).

We use a list $L$ whose elements represent pending events to be treated at specified future time instants. Each element in this list is represented by a tuple ( $t_{-}$event, $t$, reward, arm), where the possible event types are $r$ (reward) and $a$ (action), $t$ is the time instant at which the event will happen, reward is the delayed reward value and arm is the selected/pulled arm that produced the above reward. Note that, in the case of an action event, reward $=a r m=0$.

A FIFO buffer $Q_{i}$ for each arm $i, i=\{1, \ldots, K\}$ is used to store delayed rewards; $a_{k_{\text {last }}}$ is a global variable with the last executed arm; blackbox denotes whether or not the adaptation of the QPM-D algorithm for the MAB problem proposed in [14] is considered; and the reward_scenario and delay_scenario identify the reward distribution and delay type, respectively, both described in detail for the different campaign management scenarios analyzed in Section 4.

The system state consists of both the rewards stored in buffers $Q_{i}$ and the base allocation strategy (BASE) in use. The system state remains unchanged until one of the following events occurs: the reception of a delayed reward, which is stored in the corresponding buffer $Q_{i}$, and the arm selection based on BASE, which involves a BASE update and leads to the emptying of buffers $Q_{i}$.

We consider a non-homogeneous Poisson process with intensity function $\lambda(t)$ to generate the time instants in which arms have to be selected/pulled and the respective delayed reward is generated.
In the main simulation routine, see Algorithm, we first create an empty FIFO buffer $Q_{i}$ for each arm $i, i=\{1, \ldots, K\}$, generate the time instant at which an arm has to be pulled for the first time and select the arm to be pulled on the basis of BASE.

\section{Algorithm 1 Main simulation routine.}

Data: ( $T=$ maximum number of trials; $K=$ no. of arms; $a_{k}=$ arm $k, k=\{1, \ldots, K\}, a_{k_{l a s t}}$ : last selected arm; blackbox: whether or not the blackbox adaptation is used; BASE: base allocation strategy; update_architecture: points out the update architecture, reward_scenario: refers to the reward distribution; delay_scenario: refers to the delay scenario)

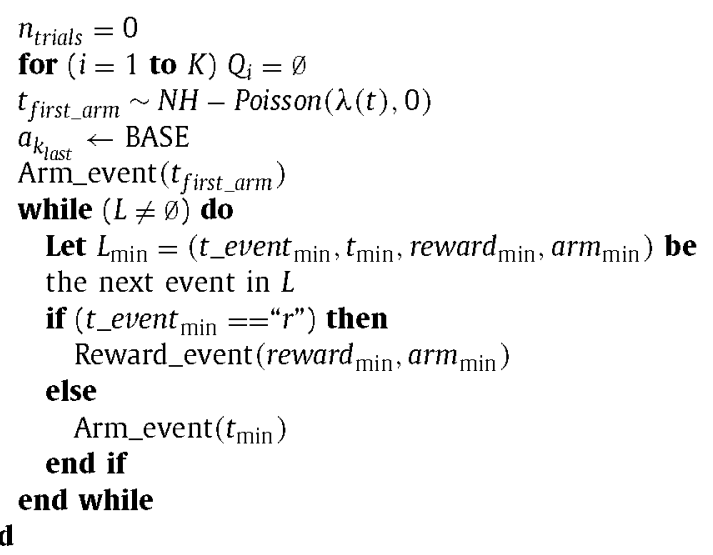

Next, we call the Arm_event function to treat this first event. Then, while the event list $L$ is not empty, we check the event that will take place next and call the respective event function to process the event. The simulation ends when $L$ is empty.

The argument in the Arm_event function is the time at which the event to be handled occurs, $t_{0}$ (see Algorithm 2).

First, the number of trials (an arm is selected) is incremented. Applying the blackbox adaptation of the base allocation strategy, we update BASE with the feedback from $Q_{k}$, including delayed rewards associated with the $\operatorname{arm} a_{k}$, select a new arm $a_{k}$ by the updated BASE and repeat the process until $Q_{k}=\emptyset$.

Applying the original allocation strategy, we just select the arm to be pulled on the basis of BASE. In this case, the current $a_{k}$ corresponds to the arm selected at time instant $t_{0}$. Thus, $a_{k_{\text {last }}}=a_{k}$. The corresponding reward $r_{t_{0}}$ is derived from the reward distribution taking into account the selected arm, and the delay $\tau$ is generated.

Finally, we store the future events in $L$. On the one hand, we store the event corresponding to the time instant at which the generated delayed reward will be received. Additionally, we have to store the event corresponding to the time instant at which a new arm selection is required. The new arm selection event will be stored in $L$ only if the number of already executed trials (arm selections) is lower than $T$.

The arguments in the Reward_event function are the delayed reward value $r$ and the respective pulled arm $k$ (see Algorithm 3). Applying the blackbox option, we add the delayed reward $r$ to the buffer $Q_{k}$. Otherwise, we update the base allocation strategy with the delayed reward value $r$.

The getDelay $\left(t_{0}\right)$ function in Algorithm can be used to account for different delay scenarios. In some cases, it outputs a fixed value $C$ throughout the algorithm iterations. However, this delay behaves stochastically in most real situations and has a different value for each time instant at which an arm is executed.

The allocation strategies shown in Table 1 will be used as BASE allocation strategies in the discrete event simulation algorithm to deal with delayed rewards. Their blackbox adaptations will be 
Algorithm 2 Arm_event $\left(t_{0}\right)$.

Data: $\left(t_{0}=\right.$ time instant at which the event occurs)

$n_{\text {trials }}++$

$a_{k}=a_{k_{\text {last }}}$

Next arm selection:

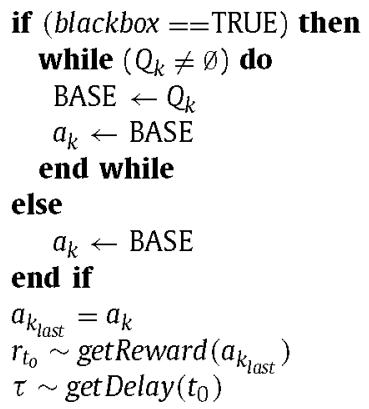

Store future events:

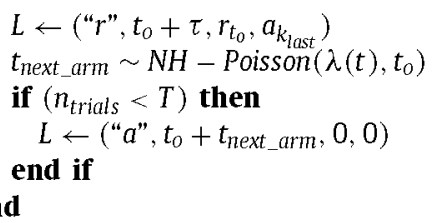

Algorithm 3 Reward_event $(r, k)$.

Data: ( $r$ : delayed reward value, $k$ : executed arm)

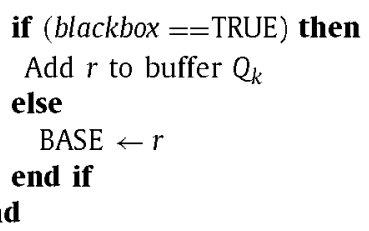

denoted by BB-UCB, BB-DMED, BB-KL-UCB, BB-KL-UCB+, BB-BESA, BB-TS, BB-PR1 and BB-PR3, respectively.

\section{Digital marketing content recommendation systems}

There are a wide variety of services in digital marketing used to offer personalized content to customers using the web, smartphones, social networks or email, such as targeted advertisement, used to show segmented advertising on the Internet depending on user profiles or their search patterns; content recommendation, used to suggest contents to a user based on his/her profile and behavior in the digital world; campaign management, aimed at personalizing the contents of a company based on previous segmentation via email, social networks, or when the user accesses the company homepage; and $A / B$ test, used to perform controlled online experimentation, evaluating the improvement of new or modifications of existing contents.

In this paper, we focused on campaign management as a scenario in the simulation process to analyze the performance of the $\mathrm{MAB}$ algorithms accounting for delayed rewards.

Campaign management solutions help marketers to create specific content (web content, banners, emails, content on social networks) that can be issued or configured to be viewed by certain segments configured according to a series of business variables, user profile or behavior. To create a campaign, a marketer has to do the following tasks:

1. Create the segment targeted by the campaign. Logical rules and variables extracted from different data sources are used for this purpose (user profile data, behavior data...). For example, a segment could be: "VIP clients who access the company's homepage more than three times a week".

2. Create the content to be offered. It could be an offer, a promotion, a message, a news item...

3. Define the channel to be used, such as the company's homepage, a social network, email, etc.

4. Define how feedback is measured and campaign success is quantified. For example, when the client clicks on a message or banner, reads an email from top to bottom, accepts a promotion...

However, a customer may set up and run several campaigns at once. Therefore, conflict management must also be configured in a similar manner to rule production in expert systems. There are several configuration modes depending on the manufacturer or the system in question: the oldest campaign, the most restrictive, apply all campaigns, prioritize all campaigns, etc.

There are not many systems that apply allow online learning in these circumstances to identify the optimal configuration conflicts between two or more campaigns. This is a scenario where algorithms reported in the literature to solve the MAB problem are clearly applicable.

There are two main types of campaign management architectures when dealing with how to update the feedback of the different contents displayed to users: batch mode and online update architectures. There are three major components in both architectures: a content manager, a content personalization engine, and one or more data sources.

The personalization process carried out in the batch mode update architecture is as follows: when a user accesses a digital channel and can receive personalized content, the manager will ask the personalization engine which content should be applied to the user. It is usual to send a parameter, such as the user identification for any context variable, together with this question.

Then, based on the configured campaigns the personalization engine queries the different data sources that apply the status of the relevant variables for the above campaigns. The content manager shows the user the content selected by the personalization engine and saves the action performed by the user when the content is displayed in its own internal database or logs.

Finally, all results are sent to the personalization engine in batch mode at certain previously configured intervals (normally on a daily basis at night. The personalization engine then evaluates the configured rewards and updates the corresponding algorithms.

In the online update architecture on the other hand, the manager queries the personalization engine when the user accesses a digital channel, and the corresponding campaign test is executed. This results in the information or reference of the content, which is displayed to the user. The content manager forwards the actions taken by the user after the content is displayed to the personalization engine. Finally, the personalization engine evaluates the configured rewards and updates the corresponding algorithms.

Note that the global update_architecture variable is used in the main simulation routine (see Algorithm 1), to denote the update architecture in use. update_architecture $=1$ means online update architecture, whereas the value 2 is used for the batch mode update architecture.

\section{Numerical analysis}

In this section, a numerical analysis will be carried out in campaign management scenarios with both online update architectures and batch mode. The arms to be selected/executed correspond to the different campaigns that can be offered to the customers. 
Table 2

Considered scenarios with an online update architecture.

\begin{tabular}{|c|c|c|c|}
\hline & Low traffic, $\lambda_{1}(t)$ & High traffic, $\lambda_{2}(t)$ & Very high traffic, $\lambda_{3}(t)$ \\
\hline Low expected rewards & Scenario 1 & Scenario 2 & Scenario 3 \\
\hline Bernoulli ( parameter $_{1}$ ) & delay $\sim[0,300]$-trunc_Exp $(1 / 80)$ & delay $\sim[0,300]-$ trunc_Exp $(1 / 80)$ & delay $\sim[0,300]$-trunc_Exp $(1 / 80)$ \\
\hline Medium expected rewards & Scenario 4 & Scenario 5 & Scenario 6 \\
\hline Bernoulli (parameter ${ }_{2}$ ) & delay $\sim[0,300]$-trunc_Exp $(1 / 80)$ & delay $\sim[0,300]-$ trunc_Exp $(1 / 80)$ & delay $\sim[0,300]$-trunc_Exp $(1 / 80)$ \\
\hline Rewards associated to delays & Scenario 7 & Scenario 8 & Scenario 9 \\
\hline Bernoulli ( parameter $_{2}$ ) & delay $\sim[0,480]$-trunc_Exp $(1 / 150)$ & delay $\sim[0,480]$-trunc_Exp $(1 / 150)$ & delay $\sim[0,480]$-trunc_Exp $(1 / 150)$ \\
\hline
\end{tabular}

Table 3

Considered scenarios with batch mode update architecture.

\begin{tabular}{|c|c|c|}
\hline & Low traffic, $\lambda_{1}(t)$ & High traffic, $\lambda_{2}(t)$ \\
\hline Low expected rewards & Scenario 10 & Scenario 11 \\
\hline Bernoulli (parameter ${ }_{1}$ ) & delay $\sim[0,300]$-trunc_Exp $(1 / 80)$ & delay $\sim[0,300]-$ trunc_Exp $(1 / 80)$ \\
\hline Medium expected rewards & Scenario 12 & Scenario 13 \\
\hline Bernoulli (parameter 2 ) & delay $\sim[0,300]-\operatorname{trunc} \_\operatorname{Exp}(1 / 80)$ & delay $\sim[0,300]-$ trunc_Exp $(1 / 80)$ \\
\hline Rewards associated to delays & Scenario 14 & Scenario 15 \\
\hline Bernoulli (parameter ${ }_{2}$ ) & delay $\sim[0,480]-\operatorname{trunc}{ }_{-} \operatorname{Exp}(1 / 150)$ & delay $\sim[0,480]-$ trunc_Exp $(1 / 150)$ \\
\hline
\end{tabular}

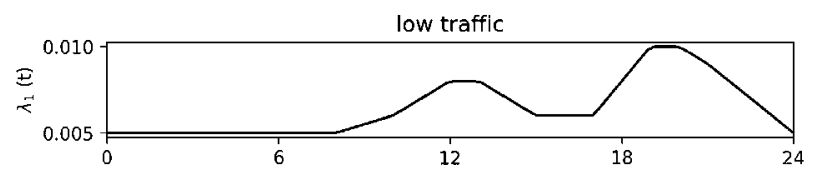

high traffic

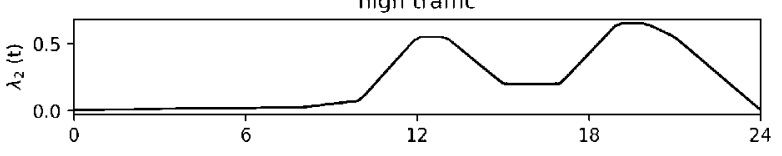

very high traffic

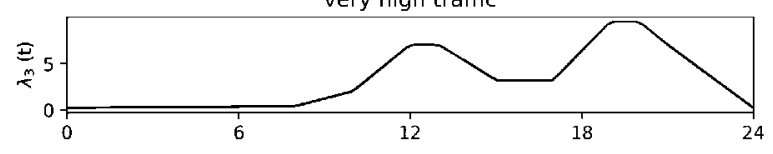

Fig. 1. Intensity functions for the web connections.

We analyze the MAB algorithms in Table 1 together with the adaptation of the QPM-D algorithm for the MAB problem which are used as the BASE algorithm. Thus, we analyze up to 16 MAB algorithms depending on the scenario under consideration.

The analyzed scenarios consist of a combination of several elements regarding campaign management. Specifically, we analyze three traffic scenarios regarding user connections to a company homepage, three reward scenarios for online and batch mode update architectures and different possible delays.

Tables 2 and 3 show all the scenarios with online and batch mode update architectures, respectively. These scenarios representing real-world campaign management are described in detail in the following sections.

\subsection{Traffic scenarios}

In our digital campaign management scenario, we consider connections to a local company homepage. The intensity functions shown in Fig. 1 represent a low $\left(\lambda_{1}(t)\right)$ a high $\left(\lambda_{2}(t)\right)$ and a very high $\left(\lambda_{3}(t)\right)$ traffic situation, respectively, regarding the non-homogeneous Poisson process used to generate the time instants at which arms have to be executed, i.e. the time instants at which the customer accesses to the company homepage.

Since we are considering local rather than global companies (with different time zones), the three intensity functions have a standard shape to account for web connections, including two time periods (morning and afternoon) where most connections are concentrated. The intensity functions are measured in connections per second.

The low traffic situation involves an average of 500 connections a day, whereas 20,000 and 250,000 connections a day are consistent with the high and very high traffic, respectively.

\subsection{Reward scenarios}

We account for three possible reward scenarios for each update architecture in the campaign management problem: a Bernoulli distribution with very low expected rewards, a Bernoulli distribution with medium expected rewards, and a variant with rewards associated with the delays.

In the first reward scenario, we use a Bernoulli distribution with very low expected rewards. This reflects a common situation in digital campaigns, where two conditions usually hold:

- Just clicking a banner, a message or a link is considered a success when the campaign is carried out on the web, mobile apps or social networks. In the case of an email-based campaign, just opening an email is also considered a success.

- The campaign target is very generic and not well segmented. Therefore, the expected success rate is normally low (about $1 \%$ ). This matches up with a common scenario in which we do not have much information about customers and their behavior towards new products and services.

For this purpose, we use the following parameter vector for the ten arms/campaigns: parameter $_{1}=$ $[0.1,0.05,0.05,0.05,0.02,0.02,0.02,0.01,0.01,0.01]$.

In the second reward scenario, the parameters are now very similar across the 10 campaigns and close to 0.5 . We assume that success conditions are the same as in the first case, but customers are now well segmented enabling quite a targeted campaign. It is unreal to simulate success rates greater than 0.5 , since even in a fine tune segmented campaign is very strange to find such high success rates are highly unlikely even in a campaign with very finely tuned segmentation.

We now use the following parameter vector for the ten arms/campaigns: parameter $_{2}=[0.5,0.45,0.45$, $0.45,0.45,0.45,0.45,0.45,0.45,0.45]$.

Finally, we simulate a scenario in the third reward scenario where the campaign content consists of a piece of news or information that should be carefully read by the user. The time that the user spends reading the news/information is a way of measuring the attention he/she pays to the content. Thus, the reward can be derived as follows: 
- First, a Bernoulli distribution is used to model the success of clicking on the news. To do this, we again consider the parameter vector in the second reward scenario, parameter ${ }_{2}$.

- If the user clicks on the news, the reward is then proportional to the time spent reading the item. A $[0,480]$ secs. truncated exponential distribution is used to generate the reading durations. The reward will be equal to 1 if the user spends $480 \mathrm{~s}$ reading the news. Otherwise, the reward is normalized taking into account the respective amount of time. The parameter $\lambda$ in the truncated exponential distribution is equal to $1 / 150$, i.e., the average reading time is $150 \mathrm{~s}$.

The getReward function used in Algorithm, which depends on the reward scenario, is shown in Algorithm.

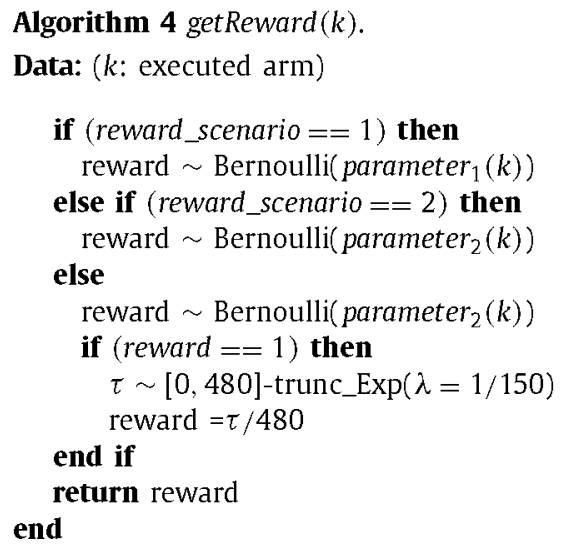

\subsection{Delay scenarios}

Different delay scenarios have also been taken into account in the getDelay function depending on the update architecture under consideration (see Algorithm 5).

In an online update architecture (update_architecture $==1$ ), we consider stochastic delays that depend on the reward scenario. If we consider rewards associated with the delays (third reward scenario), we use a $[0,480]$ truncated exponential distribution with parameter $\lambda=1 / 150$, for the reasons described above (see Algorithm 4).

Otherwise, in a Bernoulli distribution with very low or medium expected rewards, the delay is randomly generated from a $[0,300]$ truncated exponential distribution with parameter $\lambda=1 / 80$. We consider this delay distribution appropriate for digital campaigns involving the offer of up-selling, (an offer of a more beneficial service for a customer, for example a more expensive rate, greater mega consumption, etc.), cross-selling (an offer of a different service, for example, you have a bank account and you are offered home insurance) or a recommendation to use a service. This campaign type usually consists of a small text (one or two paragraphs) together with representative images of the offer. Once the customer has read the content and understood the message, he or she accepts or rejects the offer.

This delay accounts for the time it takes for the customer to receive the campaign, for it to be displayed, for the customer to read and decide whether or not to accept the offer, and for information to be subsequently returned to the campaign manager. This delay usually follows an exponential distribution, where an average expected time is usually around a mimute and a half. The delay should be truncated to a maximum ( $300 \mathrm{~s}$ ) in order to model the possibility of the customer not clicking, and to eliminate outliers.

When applying a batch mode update architecture, then the getDelay function returns the difference between 24:00 and the time instant at which the function is executed. Thus, all rewards are received at 00:00.
Algorithm 5 getDelay $\left(t_{0}\right)$.

Data: $\left(t_{0}=\right.$ time instant at which the delay is generated)

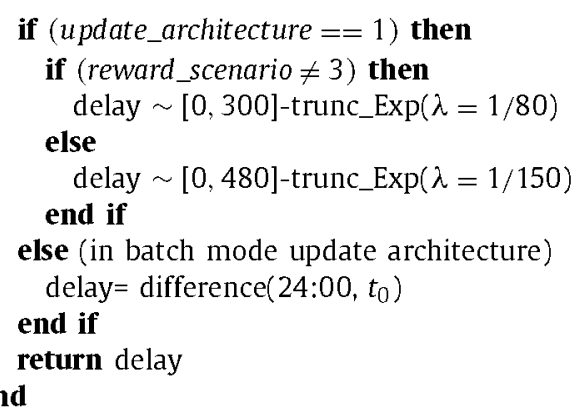

\subsection{Computational issues}

The performance of the 16 MAB algorithms under consideration will be analyzed in all the scenarios in terms of their mean cumulative regrets and standard deviations.

The mean cumulative regrets are computed as follows: a $K$-armed bandit problem can be defined by random variables $X_{i, n}$ for $1 \leq i \leq K$ and $n \geq 1$, where each $i$ is the index of one arm of a bandit and $n$ refers to the round of play. Successive plays of arm $i$ yield rewards $X_{i, 1}, X_{i, 2}, \ldots$, which are independent and identically distributed according to an unknown law with unknown expectation $\mu_{i}$.

The regret of a given allocation strategy after $n$ trials (plays) can be computed as

$\mu^{*} n-\sum_{i=1}^{K} \mu_{i} E\left[n_{i}\right]$, where $\mu^{*}=\max _{1 \leq i \leq K}\left\{\mu_{i}\right\}$,

where $\mu^{*} n$ is the total reward that can be achieved given full knowledge of the problem. E[.] denotes expectation and $n_{i}$ is the number of times arm $i$ has been played by the allocation strategy during the first $n$ plays.

Then, the mean cumulative regret after the 50,000 trials is computed using the following expression:

$\mu^{*} \times 50,000-\frac{1}{1000} \sum_{r=1}^{1000} \sum_{i=1}^{K} \sum_{j=1}^{50,000} \mu_{i} \times X_{i, j}^{r}$,

where $X_{i, j}^{r}$ denotes whether the arm $i$ is played in trial $j$ of the $r$ th simulation, and $K=10$ arms.

Note that 1000 simulations of $T=50.000$ trials (arm/campaign selections) are executed in the scenarios. The Python code available at https://github.com/mmartinb75/pyBanditsWithDelayedReward/ tree/master/pyBanditsWithDelayedReward was used for simulations, we developed any policies not implemented in the Python library, including DMED, BESA, PR-1, PR-3 and the discrete event simulation.

In simulations with an online update architecture, the average number of trials (connections) between the time an arm (campaign) is selected and the respective reward is received is $0.5,5$ and 200 for low, high and very high traffic, respectively. In the batch mode update architecture, these values are 240 and 1510 for low and high traffic, respectively.

\subsection{Campaign management with an online update architecture}

We analyze the performance of the 16 allocation strategies under consideration for the nine possible scenarios with an online update architecture and stochastic delays, see Table 2 .

Table 4 shows the resulting mean cumulative regrets and standard deviation values for the six scenarios concerning Bernoulli with very 
Table 4

Results in online update architecture with low and medium expected rewards.

\begin{tabular}{|c|c|c|c|c|c|c|}
\hline & Scenario 1 & Scenario 2 & Scenario 3 & Scenario 4 & Scenario 5 & Scenario 6 \\
\hline UCB & $602.82(87.07)$ & $611.42(85.9)$ & $633.04(76.6)$ & $742.84(150.95)$ & $744.23(169.99)$ & $780.8(151.75)$ \\
\hline BB-UCB & $602.86(84.08)$ & $605.61(85.78)$ & $627.09(78.69)$ & $715.41(159.65)$ & $725.3(155.49)$ & $753.36(159.42)$ \\
\hline DMED & $90.3(69.8)$ & $97.44(73.59)$ & $121.5(61.56)$ & $403.0(163.28)$ & $417.76(177.24)$ & $460.53(182.7)$ \\
\hline BB-DMED & $95.43(69.23)$ & $91.03(71.32)$ & $117.19(70.85)$ & $378.86(163.0)$ & $403.12(175.27)$ & $426.64(171.06)$ \\
\hline KL-UCB & $186.89(76.08)$ & $194.09(73.27)$ & $219.44(68.04)$ & $736.52(150.33)$ & $725.76(157.51)$ & $776.59(155.89)$ \\
\hline BB-KL-UCB & $191.05(66.62)$ & $192.19(72.93)$ & $213.23(73.35)$ & $718.9(158.24)$ & $727.74(150.06)$ & $744.6(151.67)$ \\
\hline KL-UCB + & $117.91(70.13)$ & $123.88(72.9)$ & $151.19(64.21)$ & $456.51(139.73)$ & $456.24(144.95)$ & $508.34(144.58)$ \\
\hline BB-KL-UCB + & $117.44(70.36)$ & $121.72(72.63)$ & $137.86(64.41)$ & $453.29(139.26)$ & $440.38(144.0)$ & $465.53(126.22)$ \\
\hline BESA & $98.74(80.12)$ & $103.03(91.35)$ & $102.01(90.53)$ & $401.83(575.82)$ & $430.49(586.63)$ & $430.14(574.81)$ \\
\hline BB-BESA & $102.32(162.47)$ & $119.48(237.1)$ & $115.93(175.96)$ & $438.64(602.83)$ & $457.85(594.14)$ & $498.72(637.67)$ \\
\hline PR1 & $294.23(74.58)$ & $285.69(71.94)$ & $291.06(78.49)$ & $361.93(160.66)$ & $337.84(142.9)$ & $366.11(147.56)$ \\
\hline BB-PR1 & $288.96(74.24)$ & $292.07(72.34)$ & $307.34(68.4)$ & $346.51(152.91)$ & $352.23(222.63)$ & $357.8(156.98)$ \\
\hline PR2 (TS) & $107.95(67.92)$ & $108.52(66.78)$ & $96.94(69.12)$ & $338.63(148.39)$ & $349.8(156.31)$ & $341.4(146.07)$ \\
\hline BB-PR2 (TS) & $111.63(64.8)$ & $106.42(69.7)$ & $113.68(65.64)$ & $342.84(157.49)$ & $347.96(144.64)$ & $359.79(167.94)$ \\
\hline
\end{tabular}

low and medium expected rewards and the three types of traffic (low, high and very high).

As pointed out in Algorithm, we randomly generate the delays from a $[0,300]$ truncated exponential with parameter $\lambda=1 / 80$.

Note that, the performance of the allocation strategy PR3 in these two reward scenarios where a Bernoulli distribution is used for the reward distribution, is exactly the same as for PR2 and Thompson sampling (TS), as pointed out in [21].

Looking at the first three columns in Table 4 (Scenarios 1-3) corresponding to a Bernoulli with very low expected rewards, we find that the performance between the original allocation strategies and their respective blackbox version is similar irrespective of the traffic. The biggest difference in the mean cumulative regret is $16.45(13.7 \%)$ for BESA in Scenario 2 (high traffic). Besides, the mean cumulative regret in some cases is greater for the original allocation strategy and lower in other cases. The standard deviations are similar for all the allocation strategies, except for BB-BESA, where they are as much as twice as high.

In Scenario 1 (low traffic), DMED and BB-DMED outperform the other allocation strategies, followed by BESA (and BB-BESA) and PR2 (and BB-PR2).

Fig. 2 shows the distribution of the accumulated regrets for the 1000 performed simulations. The allocation strategies are ordered from the one with the lowest mean cumulative regret (at the top of the graph) to the one with the highest mean cumulative regret. The best mean cumulative regret is represented by the red dotted vertical line, the mean cumulative regrets for the different allocation strategies are represented by the black dotted vertical lines, and the graph also illustrates the maximum cumulative regret for each strategy.

Looking at Fig. 2, we find that the maximum cumulative regrets for BESA and BB-BESA are quite a lot higher than for DMED, $B B-D M E D, P R 2$ and BBPR2. Besides, although the mean cumulative regret for DMED is slightly lower than for BB-DMED, the maximum cumulative regret is higher and the dispersion is also slightly greater. Therefore, both strategies could be considered the best in this scenario.

As mentioned above, PR2, PR3 and Thompson sampling are the same in these 3 scenarios.

The results are very similar with Scenario 2 (high traffic) with the exceptions that BB-DMED now outperforms DMED, and is the best allocation strategy, and PR2 (and BB-PR2) now outperforms BB-BESA (see Table 4). Looking at Fig. 3, we find that BB-MED is again the alternative with the lowest maximum cumulative regret, and the maximum cumulative regrets for BESA and BB-BESA are quite a lot higher than for the other strategies.

Finally, PR2 is the allocation strategy with the lowest mean cumulative regrets for Scenario 3 (very high traffic), followed by BESA, BB-PR2, BB-BESA (see Table 4). Looking at Fig. 4, we find that the maximum cumulative regrets for $B E S A$ and $B B-B E S A$ are again

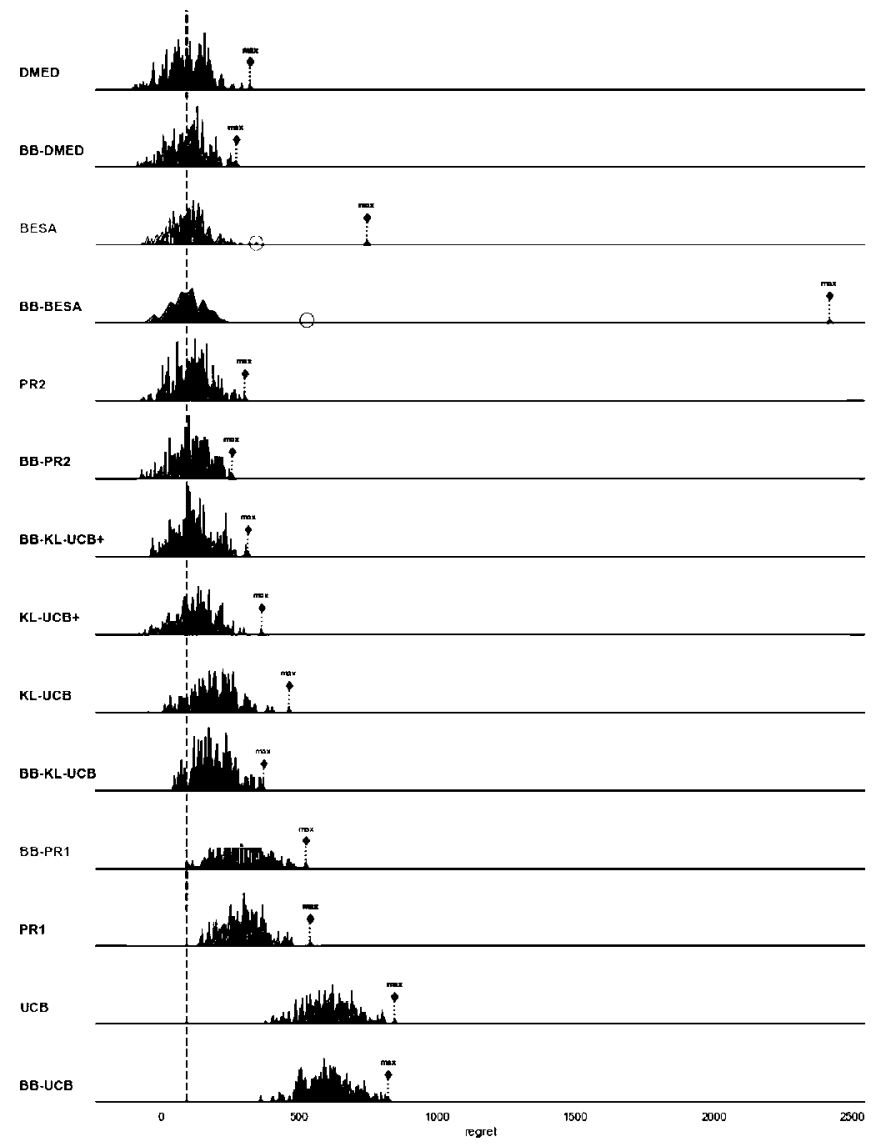

Fig. 2. Cumulative regret distribution in Scenario 1.

quite a lot higher than for the other strategies. Besides, although the mean cumulative regret for PR2 is slightly lower than for BB-PR2, the maximum cumulative regret is higher. PR2 and BB-PR2 could be considered the best strategies in this scenario.

Thus, the original DMED and BB-DMED outperform the other allocation strategies in scenario 1, where the traffic is low; BBDMED is the best for scenario 2 (high traffic), and PR2 and BB-PR2 are the best options in scenario 3 (very high traffic).

Looking at the last first three columns in Table 4 (Scenarios 4 6), corresponding to a Bernoulli with medium expected rewards, we again find that the performance of the original allocation strategies and their respective blackbox version is similar irrespective of the traffic and the mean cumulative regret is in some cases 


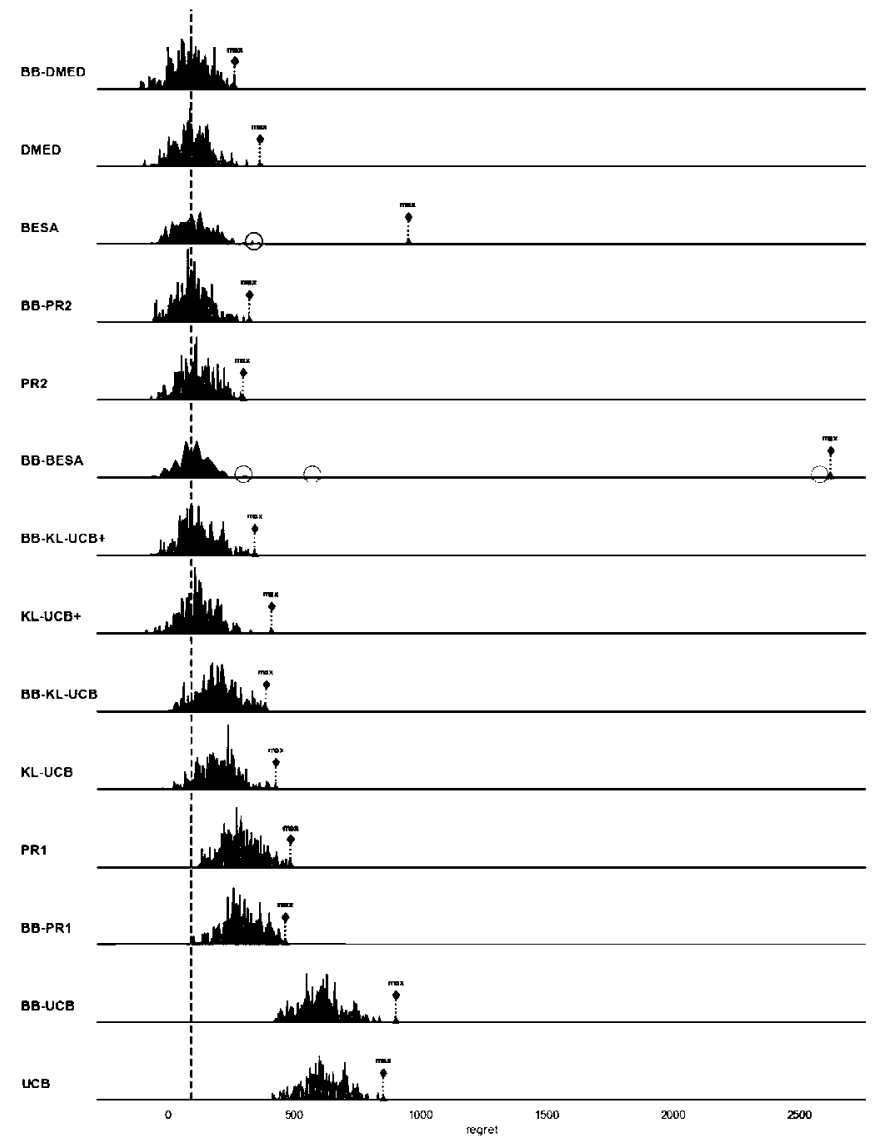

Fig. 3. Cumulative regret distribution in Scenario 2.

higher for the original allocation strategy although the opposite applies in others. The biggest difference in the mean cumulative regrets is 68.58 (13.7\%) for BESA in scenario 6 (very high traffic). The standard deviations are similar for all the allocation strategies, except for BESA and BB-BESA, which are as much as six times greater.

In Scenario 4 scenario (low traffic), PR2 and BB-PR2 outperform the other allocation strategies, followed by BB-PR1 (and PR1), BESA and BB-DMED. However, looking at Fig. 5, we find that the maximum cumulative regret for $B B-P R 2$ is higher than for PR2. Besides, BB-PR1 has both a mean and maximum cumulative regret that is slightly higher than PR2. Therefore, we can consider PR2 as the best allocation strategy followed by BB-PR1.

In Scenario 5 scenario (high traffic), PR1 is the best allocation strategy, followed by BB-PR2, PR2 and BB-PR1, with similar performances, and further behind by BB-DMED and DMED. However, looking at Fig. 6, we find that the maximum cumulative regret for PR1 is greater than for $B B-P R 2$, which is higher than for PR2, where the mean cumulative regrets are very similar for the three strategies. Thus, PR1, BB-PR2 and PR2 are the best allocation strategies in Scenario 5.

Finally, PR2 outperforms the other allocation strategies in Scenario 6 (very high traffic), followed by BB-PR1, BBPR2 and PR1, with similar performances. Looking at Fig. 7, we find that the maximum cumulative regret for PR2 is slightly higher than for $B B-P R 1$, where their mean regrets are very similar. Thus, PR2 and $B B-P R 1$ are the best allocation strategies in Scenario 5.

Thus, the original PR2 and BB-PR1 methods outperform the other allocation strategies in scenarios with a Bernoulli with medium expected rewards where the traffic is low or very high (Scenarios 4 and 6), whereas $P R 1, B B-P R 2$ and $P R 2$ are the best allocation strategies when the traffic is medium (Scenario 5).

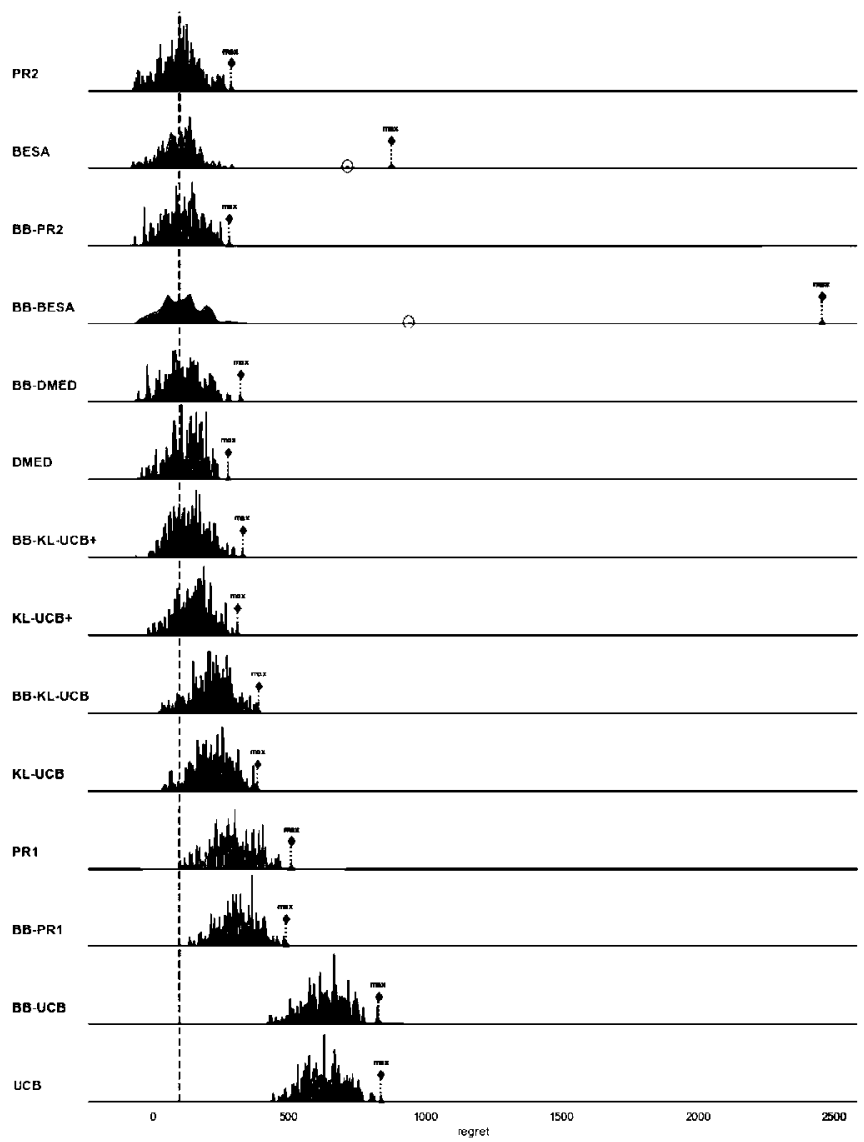

Fig. 4. Cumulative regret distribution in Scenario 3.

Table 5

Results in an online update architecture with rewards associated with the corre sponding delays.

\begin{tabular}{|c|c|c|c|}
\hline & Scenario 7 & Scenario 8 & Scenario 9 \\
\hline UCB & $1155.66(90.58)$ & $1187.47(111.35)$ & $1213.45(109.57)$ \\
\hline BB-UCB & $1153.47(90.06)$ & $1180.69(130.58)$ & $1131.56(110.77)$ \\
\hline DMED & $570.33(181.24)$ & $577.84(167.8)$ & $659.01(176.5)$ \\
\hline BB-DMED & $554.7(151.38)$ & $574.32(158.79)$ & $526.98(198.09)$ \\
\hline KL-UCB & $909.56(109.66)$ & $923.44(122.98)$ & $949.47(114.16)$ \\
\hline BB-KL-UCB & $906.81(108.16)$ & $920.09(120.6)$ & $699.26(126.9)$ \\
\hline $\mathrm{KL}-\mathrm{UCB}+$ & $624.65(127.73)$ & $627.52(117.47)$ & $644.45(120.14)$ \\
\hline BB-KL-UCB+ & $617.63(112.37)$ & $609.73(123.61)$ & $494.98(167.5)$ \\
\hline BESA & $376.86(489.9)$ & $358.56(487.99)$ & $305.72(350.99)$ \\
\hline BB-BESA & $1007.88(756.96)$ & $487.9(608.52)$ & $1087.91(808.51)$ \\
\hline PR1 & $708.87(130.97)$ & $715.52(132.34)$ & $738.45(132.06)$ \\
\hline BB-PR1 & $692.21(119.06)$ & $701.49(120.05)$ & $741.08(130.38)$ \\
\hline PR2 & $448.18(115.41)$ & $462.3(127.19)$ & $506.55(120.86)$ \\
\hline BB-PR2 & $432.4(98.41)$ & $442.82(120.97)$ & $470.29(158.54)$ \\
\hline PR3 & $345.86(115.2)$ & $353.91(106.18)$ & $396.41(113.98)$ \\
\hline BB-PR3 & $335.58(102.12)$ & $349.21(99.72)$ & $349.97(120.68)$ \\
\hline
\end{tabular}

Table 5 shows the resulting mean cumulative regrets and standard deviation values for 120,000 trials in the third reward scenario, concerning the variant with rewards associated with the corresponding delays, where the delays are randomly generated from a $[0,480]$ truncated exponential distribution with $\lambda=1 / 150$.

BB-PR3, PR3 and BESA are the best three allocation strategies, irrespective of the traffic under consideration.

Blackbox versions tend to outperform the original allocation strategies in Scenario 7 (low traffic), except for BESA, which is clearly better than BB-BESA.

Fig. 8 shows the evolution of the mean cumulative regret across trials. We can see that BESA is the best allocation strategy 


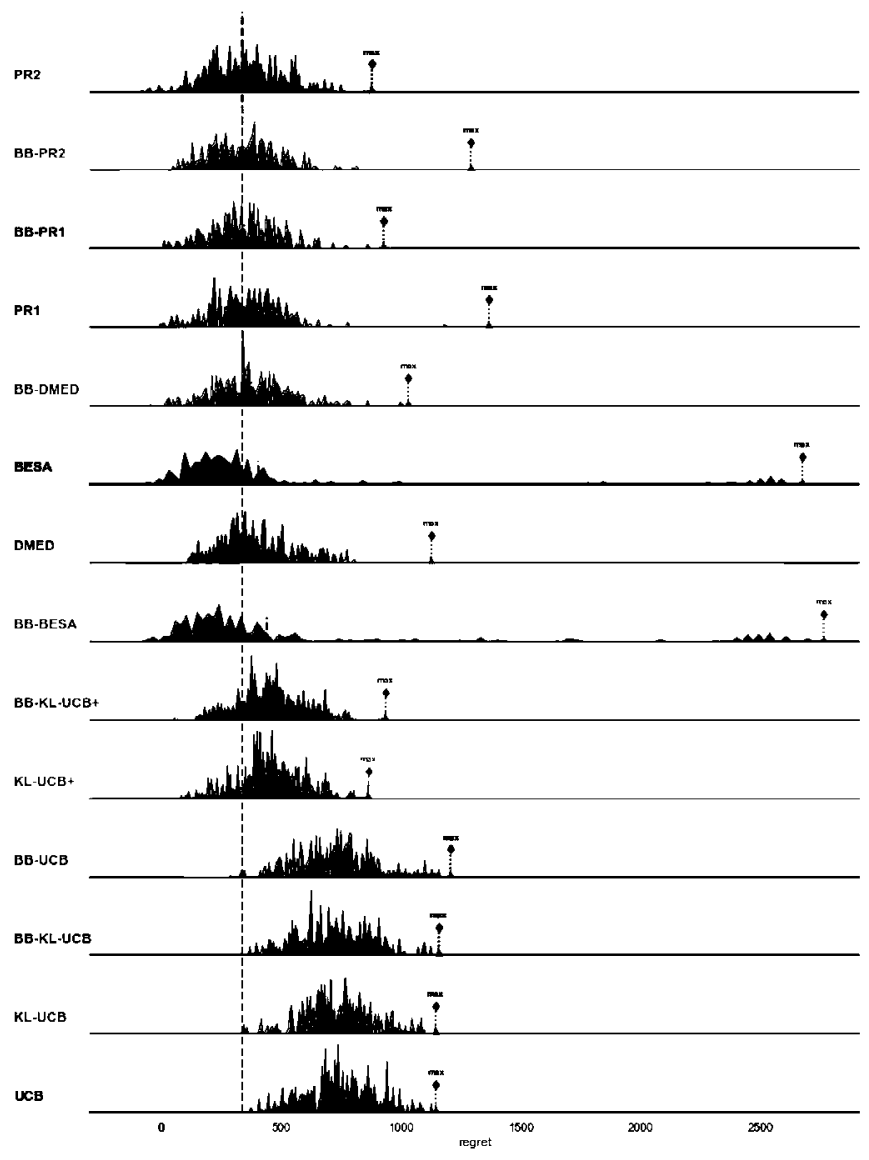

Fig. 5. Cumulative regret distribution in Scenario 4.

in terms of the mean cumulative regret when the number of trials is lower than 83,700 . For a higher number of trials, BB-PR3 is the best allocation strategy. PR3 is the second best strategy from trial 95,000 onwards. As the average number of trials per day is 500 , which is equivalent to low traffic, BB-PR3 would outperform BESA from day 175 onwards. However, the usual duration of campaigns is from 5-20 days. In this case, BESA is definitely the best allocation strategy when traffic is low. However, BB-PP3 would be better in long-term campaigns. However, it is also important to note that the standard deviation for BESA is about four times greater than for PR3 and BB-PR3. Looking at Fig. 9, we find that the maximum cumulative regret for BESA is very high with respect to BB-PR3 and PR3. Many high cumulative regrets have been output for BESA (see the mass of points close to the maximum cumulative regret). The maximum cumulative regret is very similar for $B B-P R 3$ and $P R 3$, where their mean cumulative regrets are very similar as well. Thus, BB-PR3 and PR3 are the best strategies in Scenario 7.

In Scenario 8 (high traffic), blackbox versions tend to outperform the original allocation strategies without exceptions, although performances are very similar. BESA is the best allocation strategy in terms of the mean cumulative regret when the number of trials is lower than 105,000 (see Fig. 10). For a higher number of trials, BB-PR3 is the best allocation strategy. PR3 is the second best strategy from trial 112,000 onwards. As the average number of trials per day is 20,000, which is equivalent to high traffic, BB-PR3 would outperform BESA from day 5.71 onwards. Thus, BB-PR3 is definitely the best allocation strategy when traffic is high $\left(\lambda_{2}(t)\right)$.

Besides, looking at Fig. 12, we find that the results for the best three strategies is similar to the previous scenario (see Fig. 9), except that the maximum cumulative regret for PR3 is now clearly higher than for BB-PR3, where BB-PR3 is the best allocation strategy for Scenario 8.

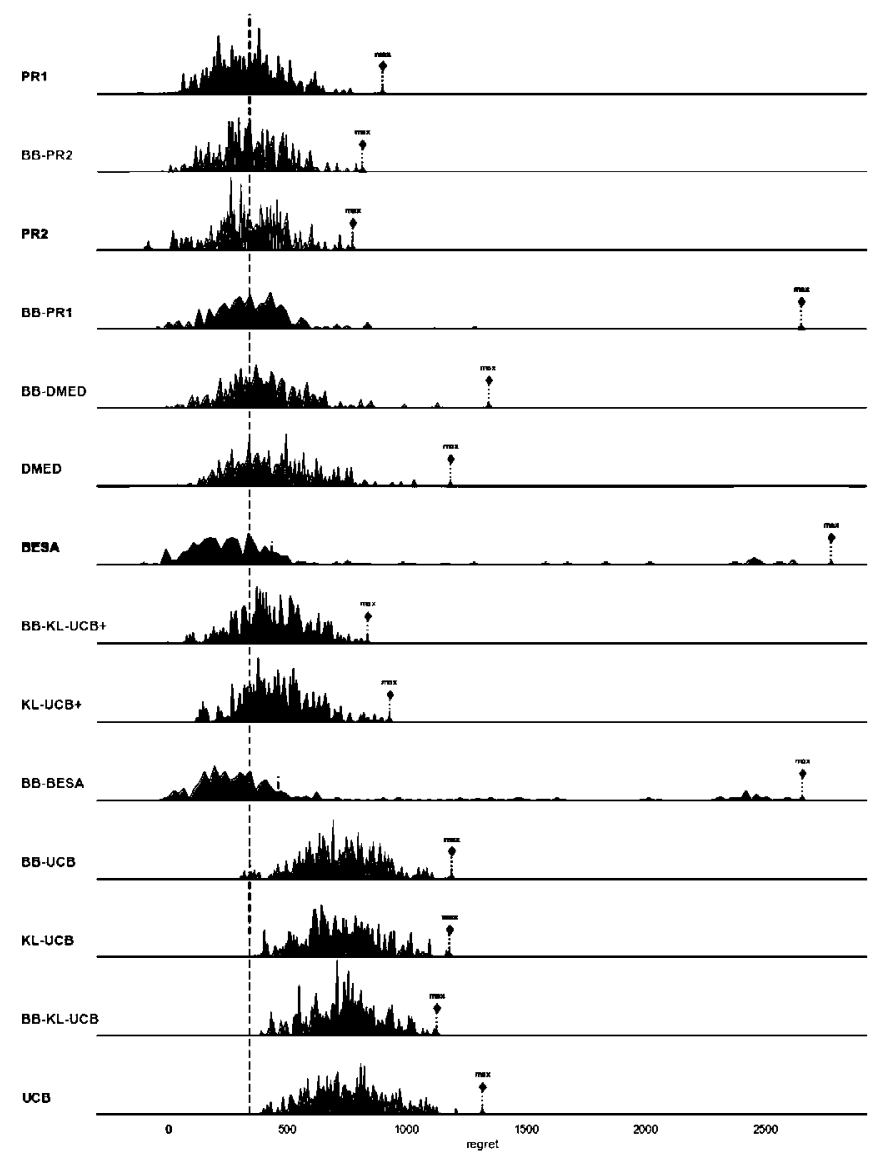

Fig. 6. Cumulative regret distribution in Scenario 5.

Finally, blackbox versions clearly outperform the respective original strategies in Scenario 9 (very high traffic), except for BESA, which is clearly better than BB-BESA. BESA is the best allocation strategy when the number of trials is lower than 358,350 (see Fig 11). For a higher number of trials, BB-PR3 is the best allocation strategy. PR3 is the second-best strategy from trial 650,000 onwards. The dotted vertical line corresponds to trial 120,000, which matches up with values in Table 5 . In high traffic, the average number of trials per day is 250,000 . Therefore, BB-PR3 would outperform BESA from day 5.71 onwards. Thus, BB-PR3 is definitely the best allocation strategy when the traffic is high $\left(\lambda_{3}(t)\right)$.

Looking at Fig. 13, we find that, although BESA outperforms the other strategies in terms of mean cumulative regrets, its maximum cumulative regret is quite high, resulting in high cumulative regrets. Besides, both the mean and maximum cumulative regrets for $B B-P R 3$ are slightly better than for PR3, where BB-PR3 is the best allocation strategy for Scenario 9. We could then conclude that BB-PR3 is the best allocation strategy in scenarios where rewards are associated with the corresponding delays (Scenarios 7-9), irrespective of the traffic under consideration.

This is highlighted by the performance of BESA in the nine scenarios with an online update architecture. Although BESA is one of the best allocation strategies in terms of the mean cumulative regret (see Scenarios 1-3, 7-9), its dispersion is in all cases higher than for the other strategies with many high cumulative regrets and also a high maximum cumulative regret.

It can be explained as follows: BESA is a stochastic algorithm based on sub-sampling without replacing the most selected arms and then selecting the arm with the best expected reward. In situations where the best expected reward is achieved for more than one sampled arm, it tends to favor exploitation by means of a follow the 


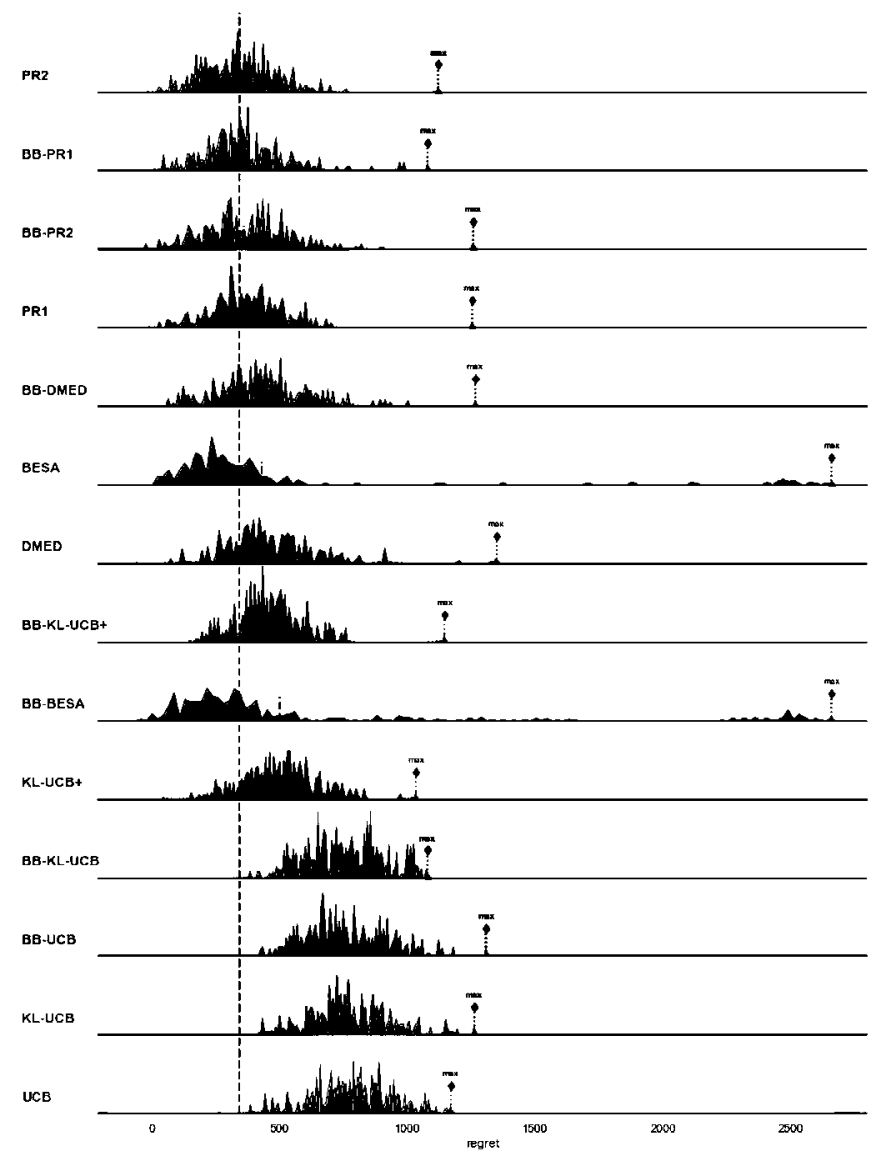

Fig. 7. Cumulative regret distribution in Scenario 6.

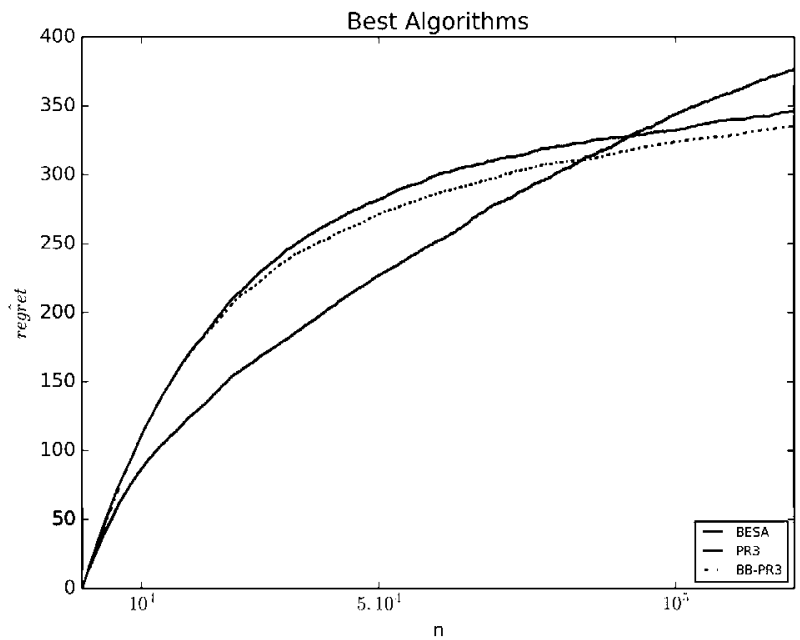

Fig. 8. Cumulative mean regret in Scenario 7 (low traffic).

leaderstrategy, i.e. selecting the arm (with the best expected rewards) that has been more often selected until then. This may main that, in an early phase of the algorithm, the selections are concentrated on a non-optimal arm that has been selected several times, leading to the dispersion of the distribution of the cumulative regrets.

\subsection{Campaign management with batch mode update architecture}

We now assume that the rewards associated with the actions performed by the users when the contents are displayed in the campaign management system are analyzed at 0:00 each day.

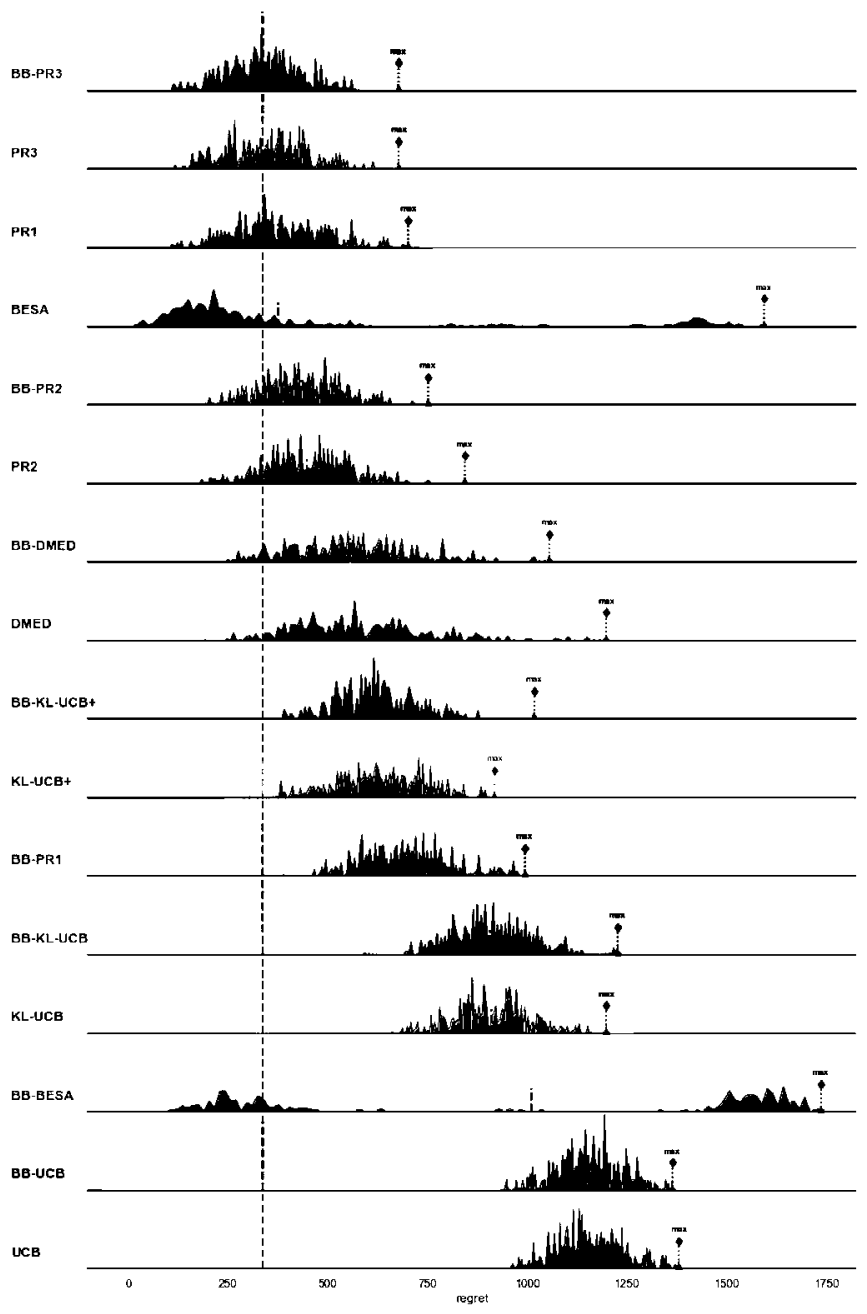

Fig. 9. Cumulative regret distribution in Scenario 7.

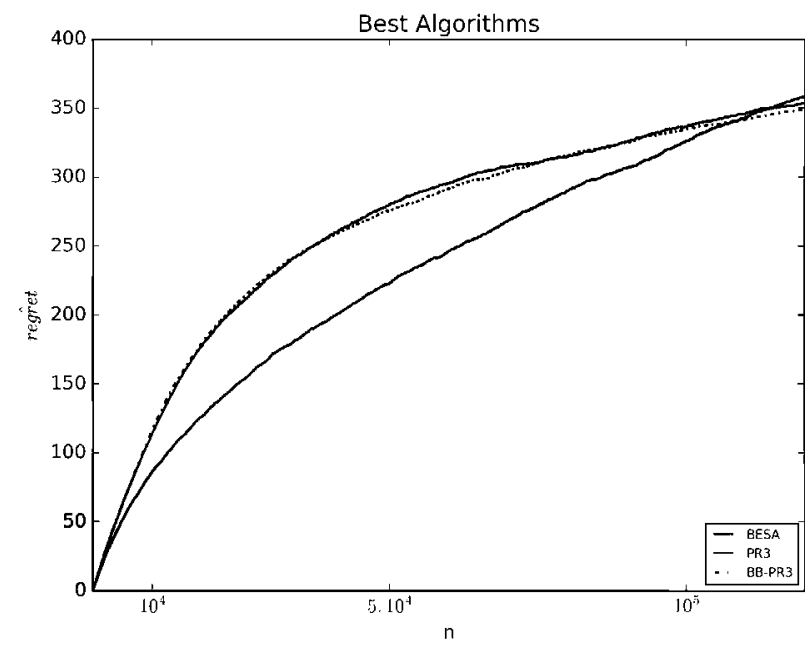

Fig. 10. Cumulative mean regret in Scenario 8 (high traffic).

Table 6 shows the mean cumulative regrets and standard deviation values for the regret after 120,000 trials using the 16 allocation strategies under consideration for the six possible scenarios with a batch mode update architecture (see Table 3).

BB-DMED outperforms DMED in the scenarios under consideration, except for Scenario 10 (low expected rewards and low traffic), 
Table 6

Results in batch mode update architecture.

\begin{tabular}{|c|c|c|c|c|c|c|}
\hline & Scenario 10 & Scenario 11 & Scenario 12 & Scenario 13 & Scenario 14 & Scenario 15 \\
\hline UCB & $817.03(87.71)$ & $2312.88(77.96)$ & $874.81(167.24)$ & $1589.23(118.42)$ & $599.41(58.81)$ & $618.01(55.6)$ \\
\hline BB-UCB & $593.92(130.28)$ & $2510.9(435.45)$ & $1249.5(1122.55)$ & $1737.9(438.82)$ & $400.76(317.49)$ & $532.92(138.96)$ \\
\hline DMED & $788.03(75.99)$ & $3267.09(46.82)$ & $1828.35(348.07)$ & $2350.95(116.23)$ & $698.75(55.66)$ & $704.97(50.16)$ \\
\hline BB-DMED & $1458.49(1362.05)$ & $2662.07(587.96)$ & $1109.94(1075.93)$ & $1736.9(422.98)$ & $343.4(310.11)$ & $608.65(127.26)$ \\
\hline KL-UCB & $429.68(71.46)$ & $721.88(152.07)$ & $872.93(168.15)$ & $1589.32(118.38)$ & $541.98(68.51)$ & $584.41(60.32)$ \\
\hline BB-KL-UCB & $1315.26(1106.14)$ & $2604.75(561.64)$ & $1249.5(1122.55)$ & $1737.9(438.82)$ & $377.41(336.54)$ & $531.55(128.41)$ \\
\hline $\mathrm{KL}-\mathrm{UCB}+$ & $388.12(66.51)$ & $536.73(144.65)$ & $606.47(149.87)$ & $1517.61(154.81)$ & $446.31(72.74)$ & $536.1(62.3)$ \\
\hline $\mathrm{BB}-\mathrm{KL}-\mathrm{UCB}+$ & $1567.88(1349.76)$ & $2604.75(561.64)$ & $1249.5(1122.55)$ & $1737.9(438.82)$ & $414.84(333.58)$ & $509.77(133.38)$ \\
\hline BESA & $96.69(64.68)$ & $218.69(72.02)$ & $390.69(436.57)$ & $427.38(251.0)$ & $205.18(180.23)$ & $229.4(152.62)$ \\
\hline BB-BESA & $1565.36(1404.33)$ & $2647.46(586.29)$ & $1369.22(1108.85)$ & $1730.14(438.33)$ & $360.19(335.46)$ & $529.49(132.0)$ \\
\hline PR1 & $289.1(80.17)$ & $324.28(75.09)$ & $349.1(140.21)$ & $405.93(147.98)$ & $452.57(78.48)$ & $464.19(77.12)$ \\
\hline BB-PR1 & $435.9(122.62)$ & $2485.55(428.93)$ & $1362.33(1112.65)$ & $1804.48(442.98)$ & $499.37(321.03)$ & $561.61(143.12)$ \\
\hline PR2 (TS) & $107.72(71.54)$ & $229.59(72.41)$ & $351.06(155.5)$ & $391.92(145.55)$ & $347.52(88.38)$ & $352.61(90.7)$ \\
\hline BB-PR2 (TS) & $1592.01(1263.46)$ & $2668.51(573.88)$ & $1413.95(1131.51)$ & $1752.21(431.29)$ & $410.44(337.29)$ & $531.98(143.3)$ \\
\hline PR3 & - & - & - & - & $288.15(86.72)$ & $297.76(88.83)$ \\
\hline BB-PR3 & - & - & - & - & $471.88(338.07)$ & $538.77(143.96)$ \\
\hline
\end{tabular}

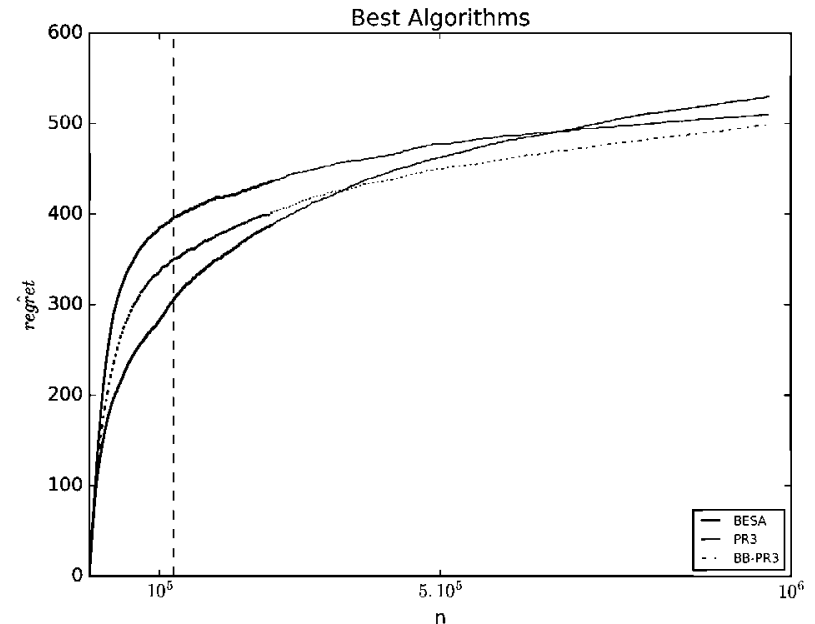

Fig. 11. Cumulative mean regret in Scenario 9 (very high traffic).

whereas the original allocation strategies outperform the blackbox version of the BESA and PR methods in all scenarios. KL-UCB+ outperforms its blackbox extension in scenarios with a Bernoulli distribution with very low and medium expected rewards (Scenarios 10-13), whereas the opposite applies to Scenarios 14 and 15.

BESA is the best allocation strategy in Scenario 10, followed closely by PR2 and further by PR1. Fig. 17 shows that BESA outperforms PR2 irrespective of the number of trials under consideration in Scenario 10.

Fig. 15 shows that the performance for BESA and PR2 is very similar, and both could be considered the best in this scenario, where BESA is ever so slightly better than PR2.

We have similar results for Scenario 11. BESA is again the best allocation strategy in terms of mean cumulative regrets, followed closely by PR2 and then by PR1. However, looking at Fig. 16, we find that the maximum cumulative regret of PR2 is clearly lower than for BESA, making PR2 the best strategy for Scenario 11.

Considering a Bernoulli with medium expected rewards, PR1 and PR2 are the best strategies with a very similar mean cumulative regret in Scenario 12 (low traffic). They are followed by BESA. However, looking at Fig. 17, we find that BESA is the best allocation strategy for a number of trials lower than 29,630. As the average number of trials with high traffic is 20,000 per day, PR1 and PR2 outperform BESA from day 1.45 onwards.

Moreover, looking at Fig. 18, we find that the maximum cumulative regret for BESA is again very high, resulting in many high cumulative regret values. Besides, the performance of $P R 1$ and $P R 2$ are very

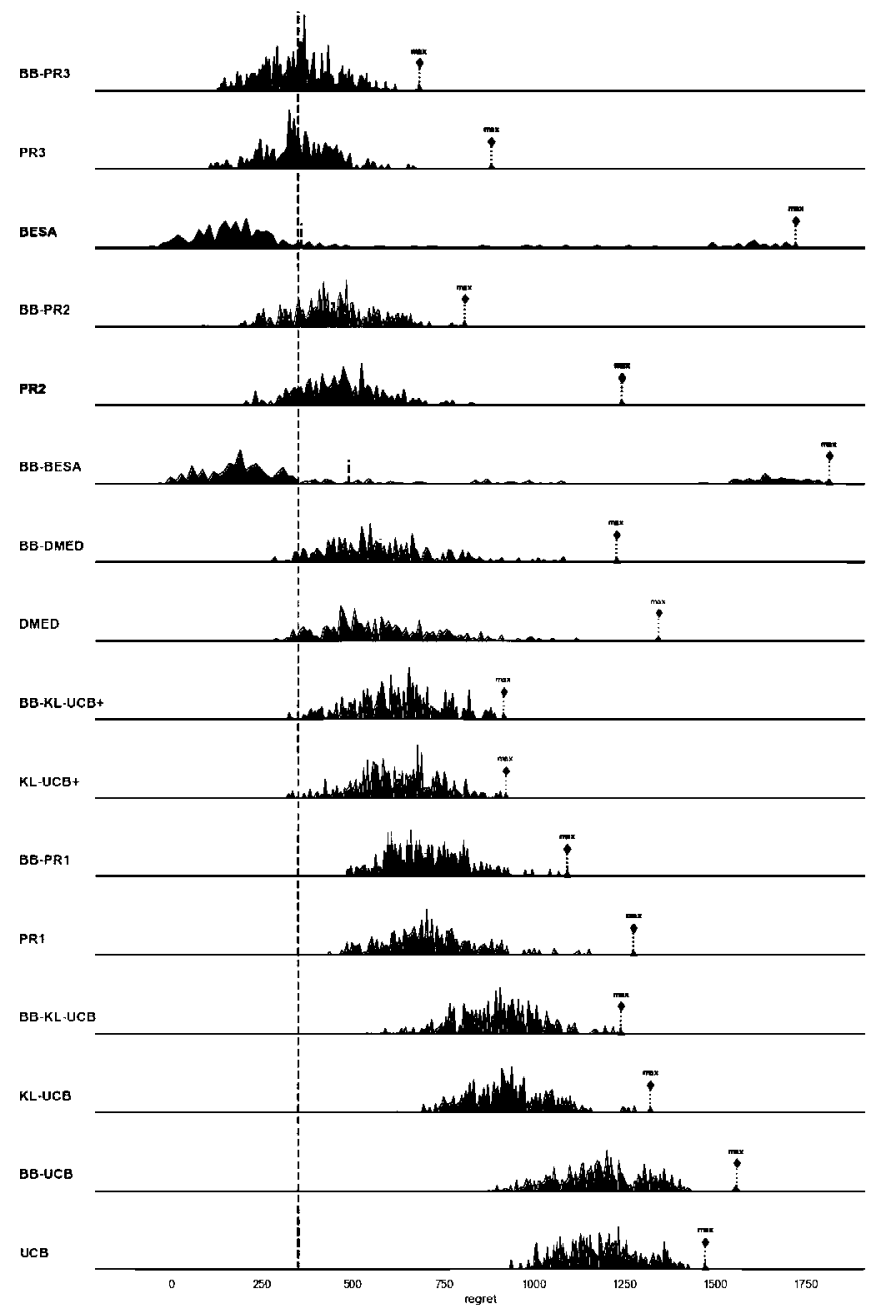

Fig. 12. Cumulative regret distribution in Scenario 8 .

similar, where PR1 is slightly better than PR2 in terms of both the mean and maximum cumulative regrets. Thus, PR1 and PR2 are the best allocation strategies in Scenario 12 .

PR2 and PR1 are again the best allocation strategies for Scenario 13, but now PR2 slightly outperforms PR1 (see Table 6 and Fig. 19).

We can conclude that PR2 and PR1 are the best allocation strategies in the batch mode architecture with medium expected rewards (Scenarios 12 and 13). 


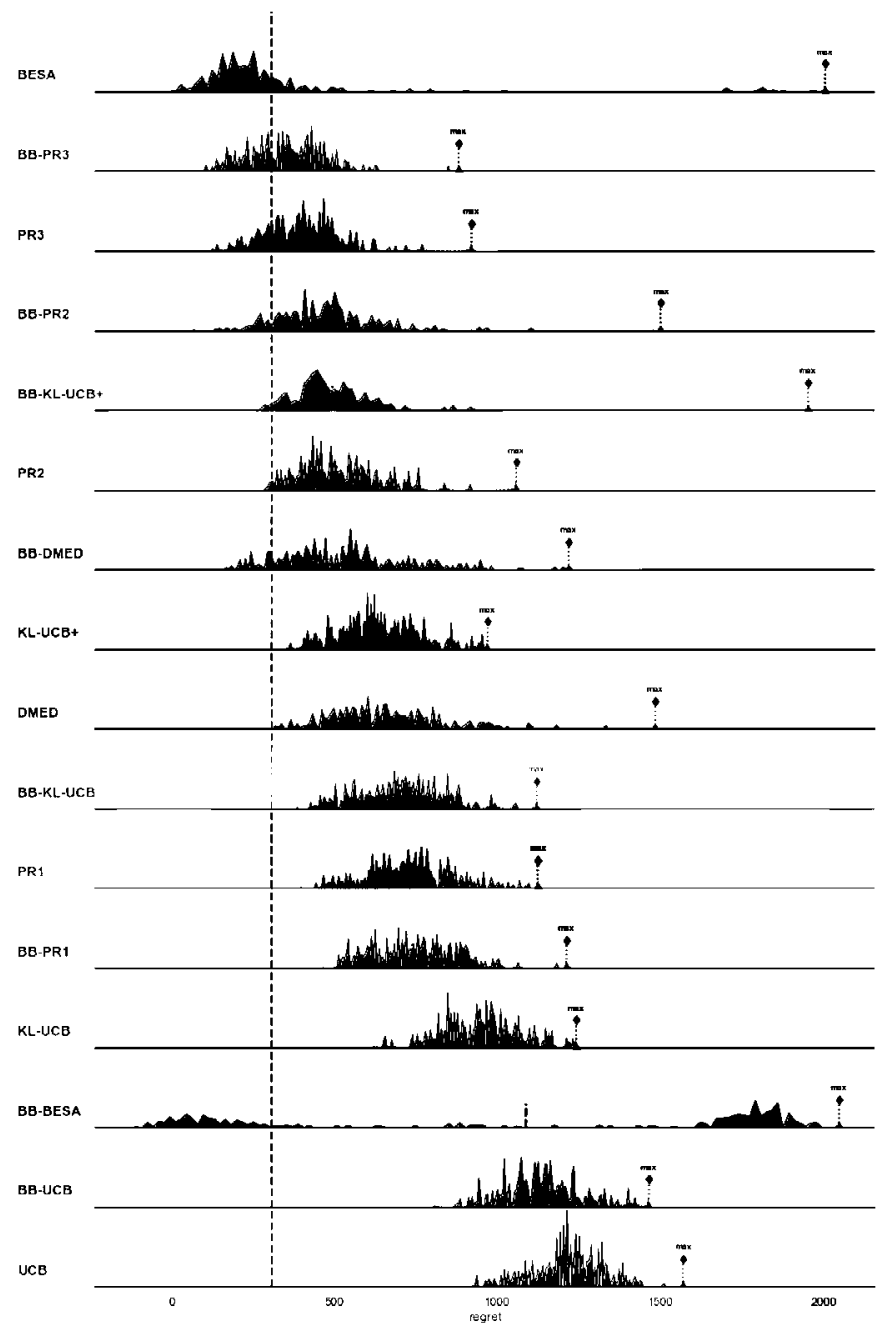

Fig. 13. Cumulative regret distribution in Scenario 9.

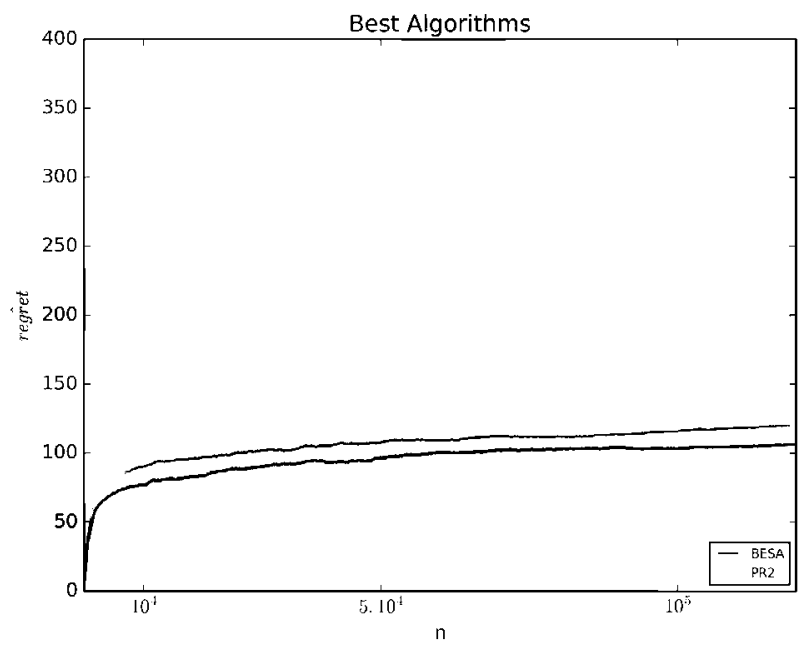

Fig. 14. Cumulative mean regret in Scenario 10 using BESA and PR2.

Finally, in Scenarios 14 and 15, the variant with rewards associated with the corresponding delays, BESA is the best strategy in terms of the cumulative mean regret for both low and high traffic irrespective of the number of trials, followed by PR3 and further by PR2. However, looking at Figs. 20 and 21, we find that in both scenarios the maximum cumulative regret for BESA is much higher

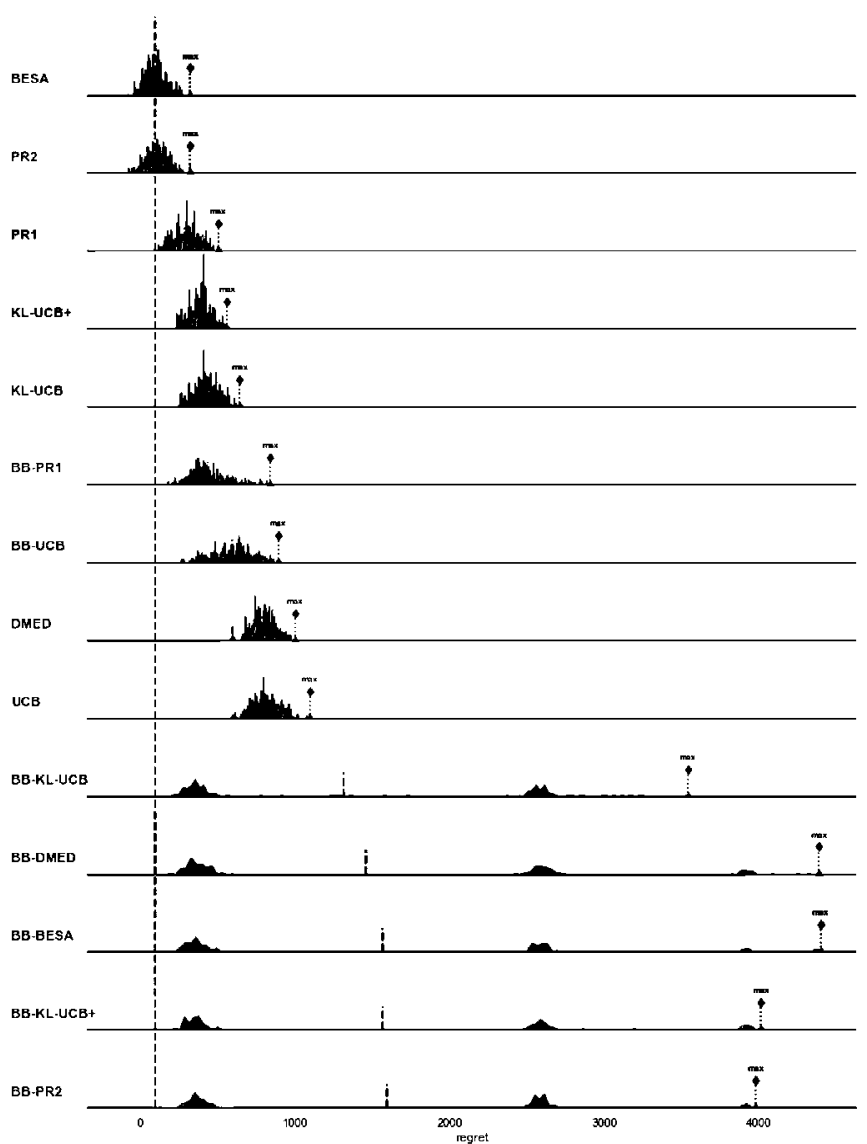

Fig. 15. Cumulative regret distribution in Scenario 10.

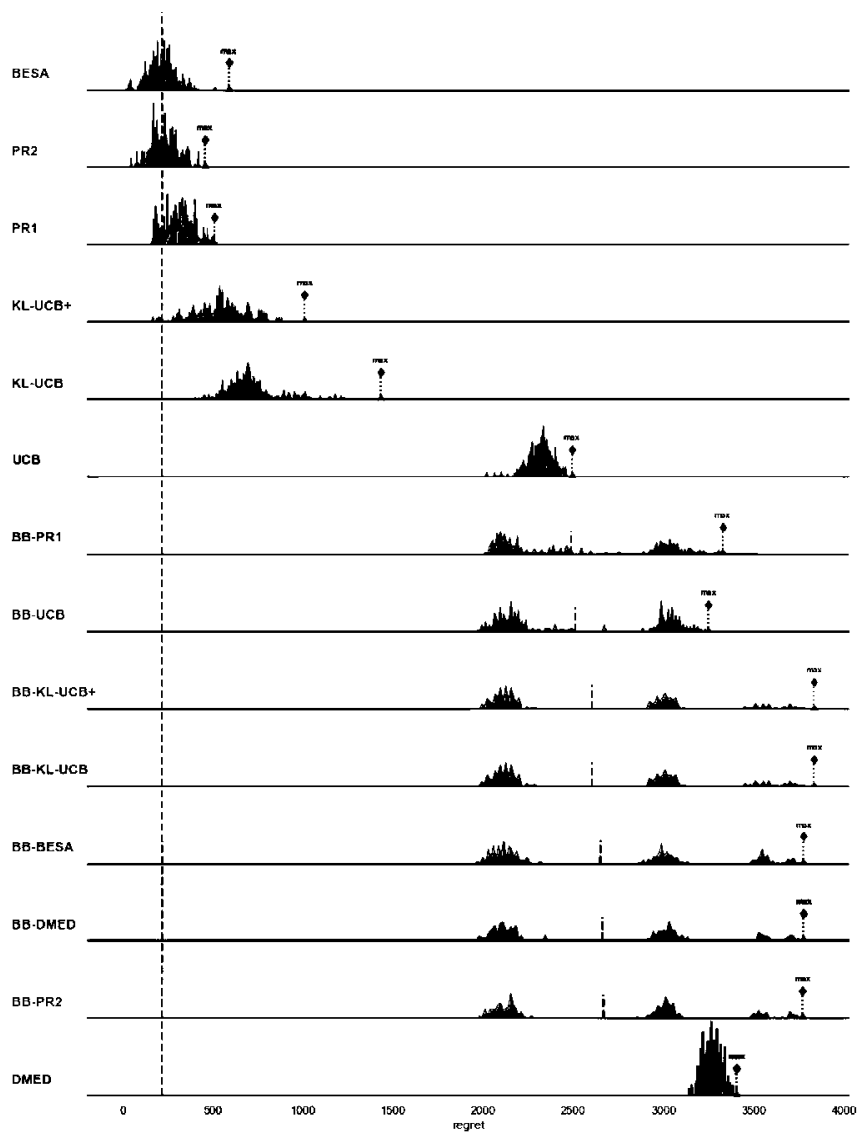

Fig. 16. Cumulative regret distribution in Scenario 11. 


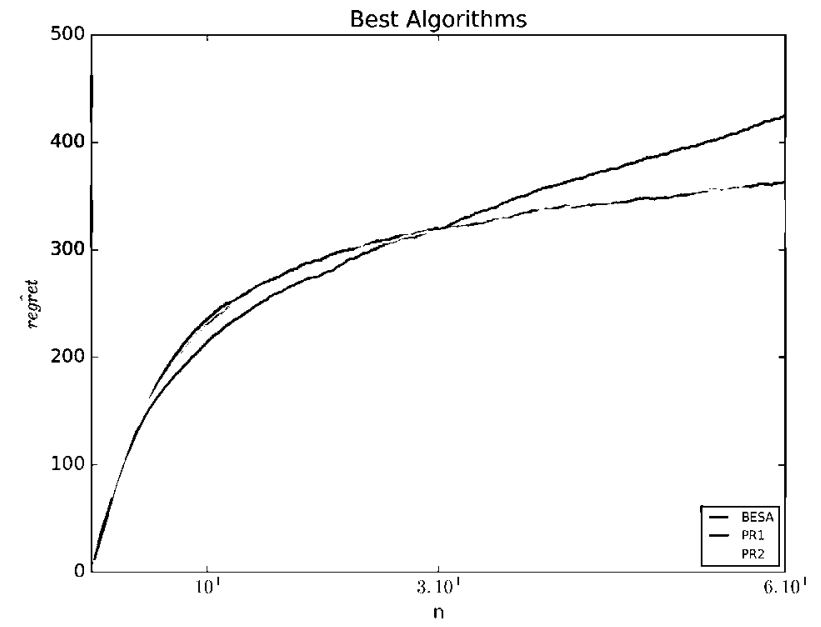

Fig. 17. Cumulative mean regret in Scenario 12 using BESA, PR1 and PR2.

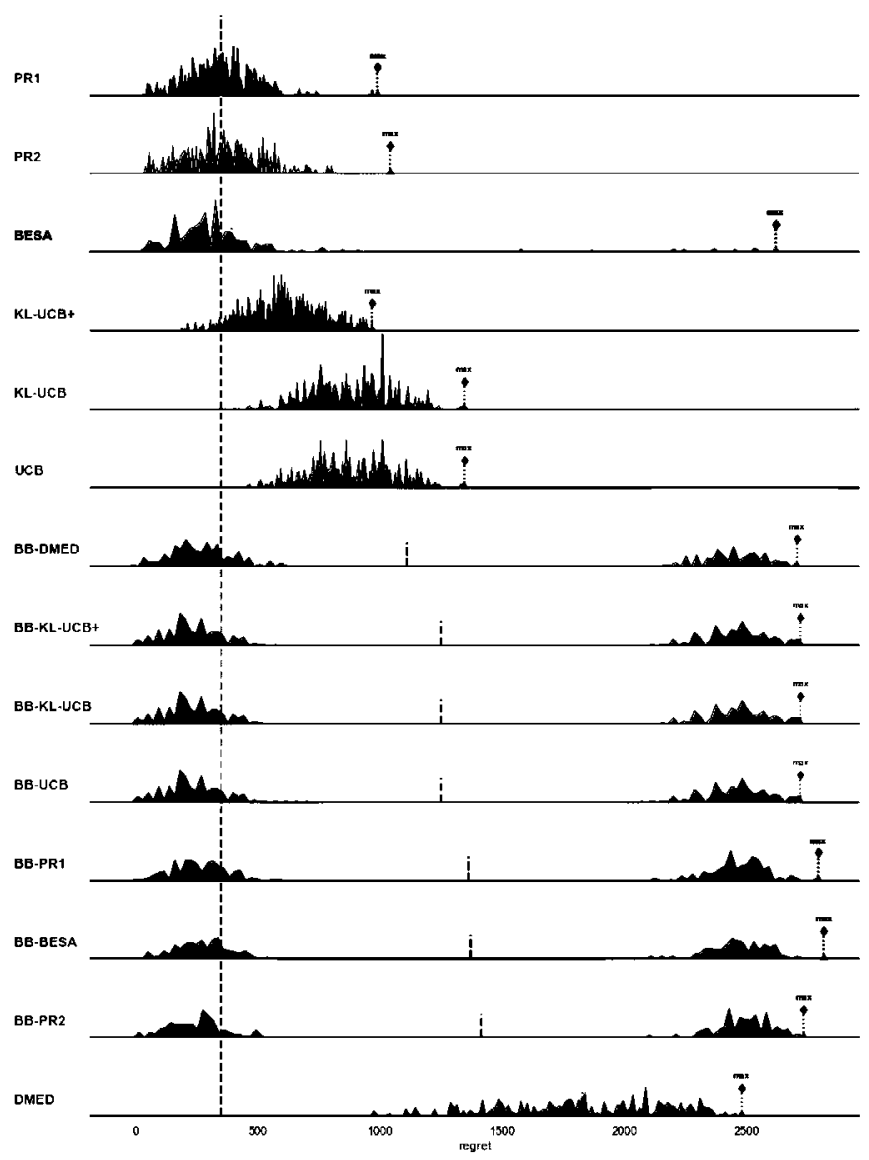

Fig. 18. Cumulative regret distribution in Scenario 12.

than for PR3. However, BESA clearly outperforms PR3 in terms of the mean cumulative regrets. Thus, it is not clear which is the best allocation strategy. If we adopt a conservative position, then PR3 is the best strategy, otherwise, BESA would be the best one.

Thus, we can conclude that the BESA and PR methods are the best allocation strategies for campaign management with a batch mode update architecture.

This is highlighted by the performance of the blackbox version of the allocation strategies with a batch mode update architecture, whose performances are worse than the respective original strategies and never are one of the best strategies. Moreover, a multi-modal cumulative regret distribution is associated with all blackbox version strategies.

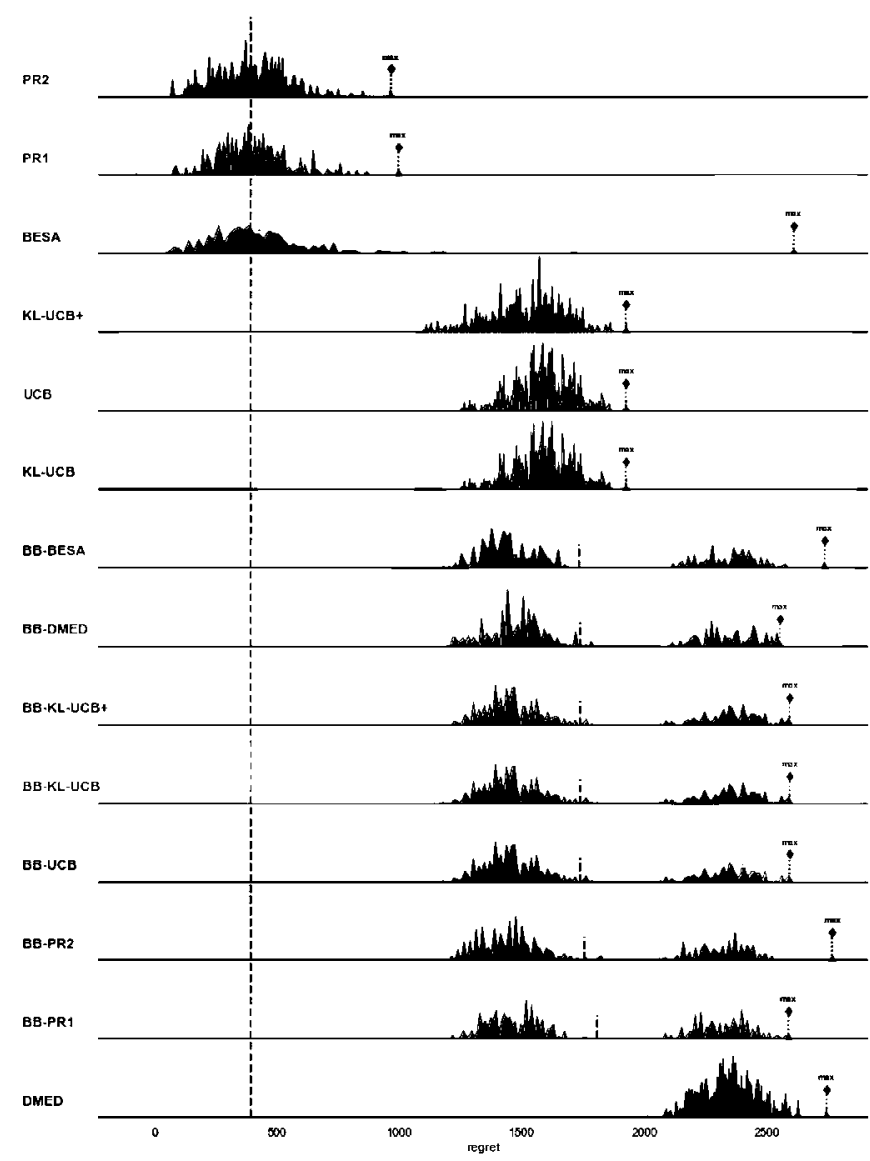

Fig. 19. Cumulative regret distribution in Scenario 13.

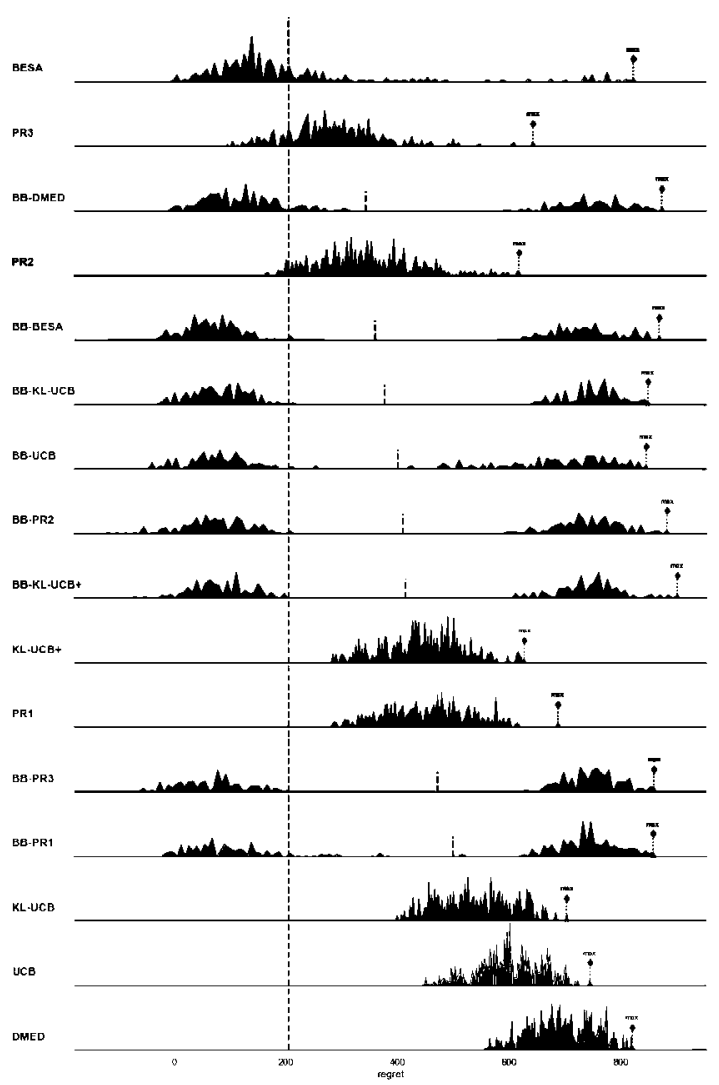

Fig. 20. Cumulative regret distribution in Scenario 14 . 


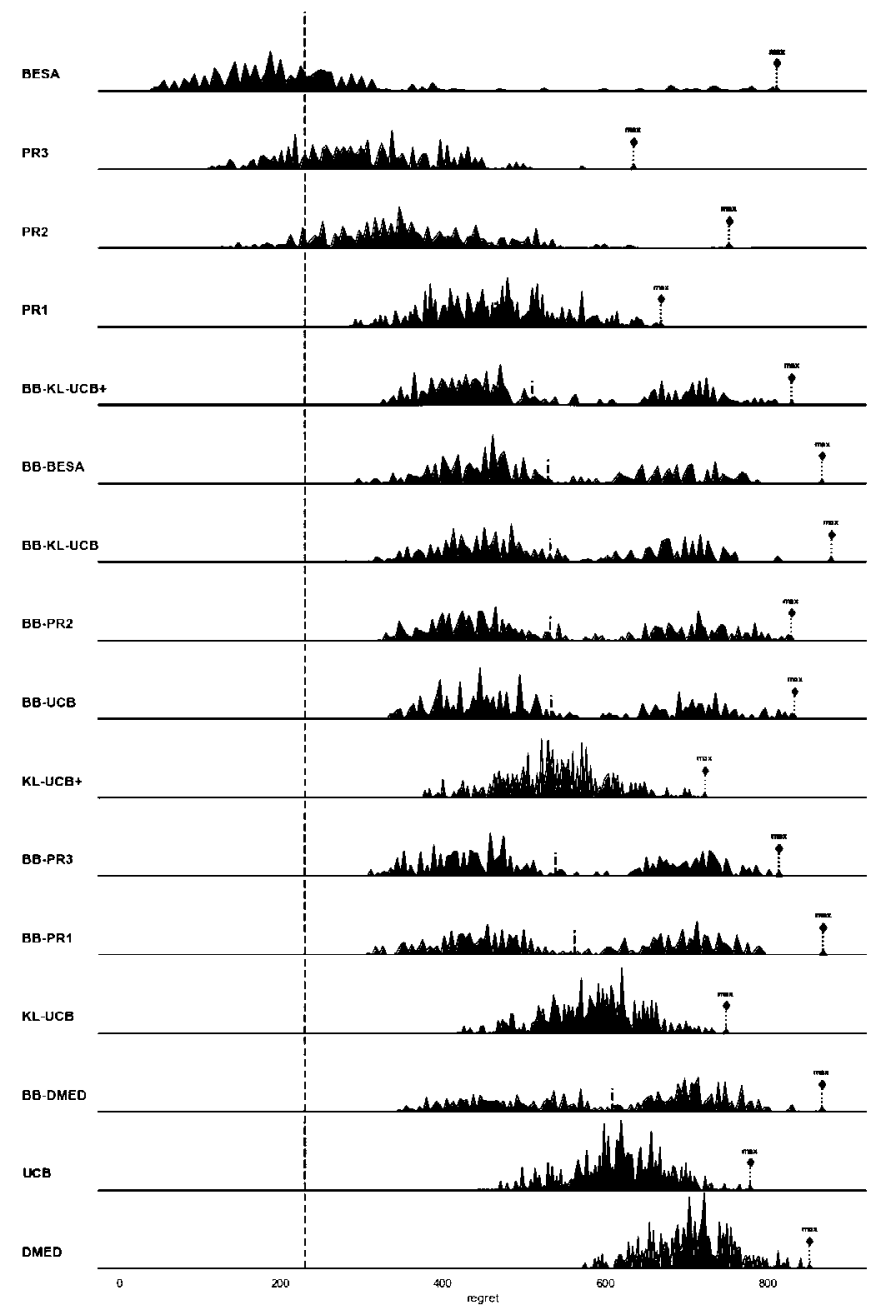

Fig. 21. Cumulative regret distribution in Scenario 15.

The explanation for the multi-modal distributions with the batch mode update architecture is the manner in which the $B B$ version encapsulates the original allocation algorithms: First, once an arm is selected, the same action will always be executed until the delayed reward is received. When traffic is low, 5000 actions (arm selections) have to be executed on average during this time, and, the same arm will be selected in all cases. This implies that at an early stage, when few rewards have been received and the models have not yet been refined, the algorithm may execute many bad actions, leading to great regrets.

The situation is worse with high traffic, where 20,000 actions have to be executed on average before the reward is received.

Note that with high traffic, the cumulative regrets are worse in Scenario 11 than in Scenario 10 (see Figs. 16 and 15). The same ap- plies for Scenarios 13 and 12 (see Figs. 19 and 18), and for Scenarios 15 and 14 (see Figs. 21 and 20), respectively.

Besides, if we compare BB algorithms in Scenarios 10 and 11 against Scenarios 12 and 13, we find that, in Scenarios 10 and 11, we have a multi-modal cumulative regret distribution, whereas in Scenarios 12 and 13, we have bi-modal distributions. This difference is associated with the number of expected reward values in the arm set in the scenarios. In Scenarios 10 and 11, there are four different expected reward values for the 10 arms under consideration $(0.1,0.05$, 0.02 , and 0.01), whereas in Scenarios 12 and 13, there are only two different expected reward values (0.5 and 0.45 ).

The explanation is as follows: in some cases, the algorithm explodes too soon, outputting a sub-optimal arm derived from a sample of rewards. Due to the behavior of the BB versions, it will take many iterations to re-exploit the best arm, leading to a mass of regrets displaced with respect to the original mass. In Scenarios 12 and 13 , with two different expected reward values, one would the best arm and the other the sub-optimal selected arm. However, in Scenarios 10 and 11 with four different expected reward values, one is the best arm, whereas the sub-optimal arm could be any of the other three expected reward values. Depending on which is the selected arm, we would have a different mass of regrets. The bigger the between the best reward and the sub-optimal selected reward, the more displaced the mass will be with respect to the original regrets but the less likely this possibility is to materialize. This explains the fact that the more displaced masses of regrets contain fewer values.

\section{Conclusions}

Table 7 shows the best allocation strategies for the scenarios, accounting for different levels of traffic and reward distributions in online and in batch update architectures.

There is no one allocation strategy that outperforms the other in all scenarios under consideration. Eight allocation strategies are the best in at least one of the 15 analyzed scenarios. PR2 is the best for seven out of 15 scenarios, followed by BB-PR3, PR3, PR1 and BB-PR2 (three scenarios), BESA and BB-DMED (two scenarios) and DMED (only one scenario). In eight cases, the blackbox version of an allocation strategy is one of the best options, whereas the original strategies (non-blackbox versions) are one of the best options in 12 out of the 15 analyzed scenarios.

Thus, depending on the configuration of the campaign management under consideration, a MAB algorithm could be the most appropriate. In an online update architecture, if a Bernoulli distribution with very low expected rewards (Scenarios $1-3$ ) is considered, then different $\mathrm{MAB}$ algorithm are proposed depending on the traffic under consideration. In a Bernoulli distribution with medium expected rewards, $P R 2$ is recommended irrespective of the traffic (Scenarios 4-6), together with BB-PR1 when the traffic is low, $B B-P R 2$ and PR1 with high traffic, and BB-PR1 with very high traffic). Finally, BB-PR3 is the best algorithm for the variant with rewards associated with the delays (Scenarios 7-9) irrespective of the traffic, together with PR3 when the traffic is medium.

Table 7

Best allocation strategies in the scenarios under consideration.

\begin{tabular}{|c|c|c|c|c|c|}
\hline & \multicolumn{3}{|c|}{ Online update architecture } & \multicolumn{2}{|c|}{ In batch mode update architecture } \\
\hline & Low traffic, $\lambda_{1}(t)$ & High traffic, $\lambda_{2}(t)$ & Very high traffic, $\lambda_{3}(t)$ & Low traffic, $\lambda_{1}(t)$ & High traffic, $\lambda_{2}(t)$ \\
\hline Low expected rewards & Scenario 1 & Scenario 2 & Scenario 3 & Scenario 10 & Scenario 11 \\
\hline Bernoulli ( parameter $_{1}$ ) & DMED \& BB-DMED & BB-DMED & PR2 \&BB-PR2 & BESA \& PR2 & PR2 \\
\hline Medium expected rewards & Scenario 4 & Scenario 5 & Scenario 6 & Scenario 12 & Scenario 13 \\
\hline Bernoulli (parameter 2 ) & PR2 \& BB-PR1 & PR1 \& BB-PR2 \& PR2 & PR2 \& BB-PR1 & PR1 \& PR2 & PR2 \& PR1 \\
\hline Rewards associated to delays & Scenario 7 & Scenario 8 & Scenario 9 & Scenario 14 & Scenario 15 \\
\hline Bernoulli (parameter 2 ) & $\mathrm{BB}-\mathrm{PR} 3$ & BB-PR3 \& PR3 & BB-PR3 & BESA \& PR3 & BESA \& PR3 \\
\hline
\end{tabular}


In a batch mode update architecture, BESA is one of the best algorithms for reward scenarios with low expected rewards and low traffic and for the variant with rewards associated with the delays, irrespective of the traffic (Scenarios 14 and 15), whereas PR2 can be used when a Bernoulli distribution with low or medium expected rewards (Scenarios 10-13) is considered. Finally, PR3 can be used together with BESA when rewards associated with the delays are taken into account (Scenarios 14 and 15), irrespective of the traffic. Note that the best strategies in a batch mode update architecture do not include the blackbox version of any of the allocation strategies.

A future research line that we propose is to extend the analysis carried out in this paper to the $A / B$ test, used to perform controlled online experimentation, evaluating the improvement of new or modifications of existing contents. There are some tools such as Google Analytics that offer an advanced A/B test solution based on $\mathrm{MAB}$ algorithms. In this future research line, the performance of these tools could be evaluated and compared with the traditional A/B test solutions.

Other interesting future evaluation scenarios are campaigns to send emails, sms or push notifications. In this case, the simulation features would differ from the ones considered in this paper. First, there is no arrival rate. It is the campaign manager who sends emails, sms or push notifications according to a sending rate ( $\mathrm{X}$ emails every $\mathrm{N}$ seconds, for example). Besides, the delay would, in this case, be measured by the time elapsed between when a customer receives and when a customer opens an email. This behavior should be modeled by means of a probability distribution also taking into account that the parameters of the distribution will vary throughout the day.

It would also be interesting to model quite a real environment, in which the reward is received through a small survey with binary (yes/no) or discrete (1-5) answers, one time instant after the content is offered to the client.

\section{Declaration of interest}

The authors declare that they have no known competing financial interests or personal relationships that could have appeared to influence the work reported in this paper.

\section{References}

[1] R. Agrawal, Sample mean based index policies with $O(\log n)$ regret for the multi-armed bandit problem, Adv. Appl. Probab. 27 (1995) 1054-1078.

[2] A. Agarwal, J. Duchi, Proceedings of the 24th International Conference on Neural Information Processing Systems 24 (2011) 873-881.

[3] S. Agrawal, N. Goyal, Analysis of thompson sampling for the multi-armed bandit problem, in: Proceedings of the Conference Learning Theory, 23, 2012. pp. $39-1,39-26$

[4] M.H.Z. Ashtiani, M.N. Ahmadabadi, B.N. Araabi, Bandit-based local feature subset selection, Neurocomputing 138 (2014) 371-382.

[5] P. Auer, N. Cesa-Bianchi, P. Fischer, Finite-time analysis of the multiarmed bandit problem, Mach. Learn. 47 (2002) 235-256.

[6] A. Baransi, O.A. Maillard, S. Mannor, Sub-sampling for multi-armed bandits, in: Proceedings of the European Conference Machine Learning, 2014, pp. 115-131.

[7] D. Berry, B. Fristedt, Bandit Problems, Chapman and Hall, London, 1985.

[8] D. Bouneffouf, R. Feraud, Multi-armed bandit problem with known trend, Neu rocomput. 205 (2016) 16-21.

[9] T. Desautels, A. Krause, J. Burdick, Parallelizing exploration-exploitation tradeoffs with gaussian process bandit optimization, J. Mach. Learn. Res. 15 (2014) 4053-4103.

[10] M. Dudik, D. Hsu, S. Kale, N. Karampatziakis, J. Langford, L. Reyzin, T. Zhang, Efficient optimal learning for contextual bandits, in: Proceedings of the 27th International Conference on Uncertainty in Artificial Intelligence, 2011, pp. $169-178$.

[11] A. Garivier, O. Cappé, The KL-UCB algorithm for bounded stochastic bandits and beyond 24 (2011) 359-376.

[12] J. Holland, Adaptation in Natural and Artificial Systems, MIT Press/Bradford Books, Cambridge, 1992

[13] J. Honda, A. Takemura, An asymptotically optimal bandit algorithm for bounded support models, in: Proceedings of the 24th Annual Conference Learning Theory, 2010, pp. 67-79.
[14] P. Joulani, A. György, C. Szepesvári, Online learning under delayed feedback, in: Proceedings of the 30th International Conference on Machine Learning, 2013.

[15] E. Kaufmann, O. Cappé, A. Garivier, On Bayesian upper confidence bounds for bandit problems, in: Proceedings of the International Conference Artificial Intelligence and Statistics, 2012, pp. 592-600.

[16] A. Lacerda, Multi-objective ranked bandits for recommender systems, Neurocomput. 246 (2017) 12-24

[17] T. Lai, H. Robbins, Asymptotically efficient adaptive allocation rules, Adv. Appl. Math. 6 (1985) 4-22.

[18] L. Li, C. W, J. Langford, R.E. Schapire, A contextual-bandit approach to personalized news article recommendation, in: Proceedings of the 19th International Conference World Wide Web, 2010, pp. 661-670.

[19] T. Lu, D. Pal, M. Pal, Contextual multi-armed bandits, in: Proceedings of the 13th International Conference Artificial Intelligence and Statistics, 2010 pp. 485-492.

[20] M. Martín, A. Jiménez-Martín, A. Mateos, Possibilistic reward method for the multi-armed bandit problem, in: Proceedings of the 6th International Conference on Operations Research and Enterprise Systems, 2017, pp. 75-84.

[21] M. Martín, A. Jiménez-Martîn, A. Mateos, The possibilistic reward methods for the multi-armed bandit problem, Neurocomput. 310 (2018) 210-212.

[22] G. Neu, A. György, C. Szepesvảri, A. Antos, Online Markov decision processes under bandit feedback, IEEE Trans. Autom. Control. 59 (2014) 676-691.

[23] C. Pike-Burke, S. Agrawal, C. Szepesvári, S. Grünewälder, Bandits with delayed, aggregated anonymous feedback, in: Proceedings of the 35th International Conference Machine Learning, 2018.

[24] R. Sutton, A. Barto, Reinforcement Learning, an Introduction, MIT Press, Cambridge, 1998.

[25] W.R. Thompson, On the likelihood that one unknown probability exceeds another in view of the evidence of two samples, Biometrika 25 (3-4) (1933) 285-294.

[26] C.C. Wang, S.R. Kulkarni, H.V. Poor, Arbitrary side observations in bandit problems, Adv. Appl. Math. 34 (2005) 903-938.

[27] Y. Xia, T. Qin, W. Ding, T.Y. Liu, Finite budget analysis of multi-armed bandit problems, Neurocomputing 258 (2017) 13-29. 